\title{
RELATIVE CYCLIC HOMOLOGY OF SQUARE ZERO EXTENSIONS
}

\author{
JORGE A. GUCCIONE AND JUAN J. GUCCIONE
}

\begin{abstract}
Let $k$ be a characteristic zero field, $C$ a $k$-algebra and $M$ a square zero two sided ideal of $C$. We obtain a new mixed complex, simpler than the canonical one, giving the Hochschild and cyclic homologies of $C$ relative to $M$. This complex resembles the canonical reduced mixed complex of an augmented algebra. We begin the study of our complex showing that it has a harmonic decomposition like to the one considered by Cuntz and Quillen for the normalized mixed complex of an algebra. We also give new proofs of two theorems of Goodwillie, obtaining a light improvement of one of them.
\end{abstract}

\section{INTRODUCTION}

Let $k$ be a characteristic zero field, $C$ a $k$-algebra and $M$ a two-sided ideal of $C$. In this work we deal with the Hochschild, cyclic, periodic and negative homologies of $C$ relative to $M$, when $M^{2}=0$. Our main result is Theorem 3.2 in which we obtain a double mixed complex $(\hat{X}, \hat{b}, \hat{d}, \hat{B})$, given these homologies, whose associated mixed complex $(\breve{X}, \breve{b}, \breve{B})$ is simpler than the canonical mixed complex of $C$ relative to $M$. We hope that $(\hat{X}, \hat{b}, \hat{d}, \hat{B})$ be useful to prove results about cyclic type homologies of an algebra relative to a nilpotent ideal, by induction on the degree of nilpotence. Evidence in such sense is provided by Theorem [4.6] in which we improve a result of Goodwillie. We also hope that $(\hat{X}, \hat{b}, \hat{d}, \hat{B})$ be a first step to obtain explicit computations of cyclic homology groups of an algebra $C$ relative to a two sided square zero ideal.

The paper is organized in the following way:

In Section 2 we recall some well known definitions and results. Among them, the perturbation lemma, which we will use again and again in the rest of the paper, and the definition of double mixed complex, which we got from $\mathrm{Co}$.

Section 3 is devoted to establishing the main results in this paper. Since $M^{2}=0$, the algebra $C$ is isomorphic to a square zero extension $E=A \ltimes_{f} M$, where $A=C / M$ and $f: A \otimes A \rightarrow M$ is a Hochschild normal 2-cocycle. So, we can restrict our attention to this type of algebras. In fact, $(\hat{X}, \hat{b}, \hat{d}, \hat{B})$ can be thought as a double mixed complex associated to the 3 -tuple $(A, M, f)$, and this association is functorial in an evident sense. For $0 \leq 2 w \leq v$, let $X_{v}^{w}$ be the direct sum of all the tensor products $X_{0} \otimes \cdots \otimes X_{n}$ such that $X_{0}=M, X_{i}=M$ for $w$ indices $i>0$ and $X_{i}=\bar{A}$ for the other ones, where $n=v-w$ and $\bar{A}=A / k$. Let $b: X_{v}^{w} \rightarrow X_{v-1}^{w}$ be the map given by the same formula as the Hochschild boundary map of an algebra, where the meaning of the concatenation $x_{i} x_{i+1}$ of two consecutive factors in a simple tensor means is the one given in item (3) of Notation 1.2 Let $t: X_{v}^{w} \rightarrow X_{v}^{w}$ be the map defined by

$$
t\left(x_{0} \otimes \cdots \otimes x_{n}\right)=(-1)^{i n} x_{i} \otimes \cdots \otimes x_{n} \otimes x_{0} \otimes \cdots \otimes x_{i-1},
$$

2000 Mathematics Subject Classification. Primary 16E40, Secondary 16S70.

Supported by PICT 12330, UBACYT 0294 and CONICET.

Supported by PICT 12330, UBACYT 0294 and CONICET. 
where $i$ denotes the last index such that $x_{i} \in M$ and let $N=\mathrm{id}+t+t^{2}+\cdots+t^{w}$. The double mixed complex $(\hat{X}, \hat{b}, \hat{d}, \hat{B})$ has objects $\hat{X}_{v}^{w}=X_{v}^{w} \oplus X_{v-1}^{w}$. The boundary maps are given by

$$
\hat{b}(\mathbf{x}, \mathbf{y})=(b(\mathbf{x})+(\text { id }-t)(\mathbf{y}),-b(\mathbf{y})) \quad \text { and } \quad \hat{d}(\mathbf{x}, \mathbf{y})=\left(d(\mathbf{x}), d^{\prime}(\mathbf{y})\right),
$$

where $d, d^{\prime}: X_{v}^{w} \rightarrow X_{v}^{w+1}$ are maps depending on $f$, and the Connes operator is given by $\hat{B}(\mathbf{x}, \mathbf{y})=(0, N(\mathbf{x}))$. So, it resembles the reduced mixed complex of an augmented algebra. Since the maps $t$ and $N$ satisfy

$$
\operatorname{Im}(1-t)=\operatorname{ker}(N) \text { and } \operatorname{Im}(N)=\operatorname{ker}(1-t),
$$

the cyclic homology of $E$ relative to $M$ is the homology of the quotient complex of $(X, b, d)$ by the image of id $-t$. Indeed, this also follows from the fact that $(\hat{X}, \hat{b}, \hat{d}, \hat{B})$ satisfies the Connes property ( $\mathrm{C}-\mathrm{Q})$, which is another consequence of the equalities (1). We finish the section giving a new proof of the following celebrate theorem of Goodwillie: if $M$ is a nilpotent two-sided ideal of a $k$-algebra $C$, then $\operatorname{HP}(C)=\operatorname{HP}(C / M)$.

The aim of Section 4 is to show that $(\breve{X}, \breve{b}, \breve{B})$ has a harmonic decomposition like the one studied in $\mathrm{C}-\mathrm{Q}$. In order to carry out this task we need to define a de Rham coboundary map and a Karoubi operator on $(\breve{X}, \breve{b})$. Actually it will be convenient for us to work with a new double mixed complex, namely $(\ddot{X}, \ddot{d}, \ddot{b}, \ddot{B})$, whose associated mixed complex is also $(\breve{X}, \breve{b}, \breve{B})$. As in C-Q the Karoubi operator $\ddot{\kappa}$ of $(\ddot{X}, \ddot{d}, \ddot{b})$ commutes with $\ddot{b}$ and $\ddot{d}$ and satisfies a polynomial equation $P_{w}(\kappa)$ on each $\ddot{X}_{v}^{w}$. Thus we have the harmonic decomposition $\ddot{X}=P(\ddot{X}) \oplus P^{\perp}(\ddot{X})$, where $P$ is the spectral projection onto the generalized nullspace for id $-\ddot{\kappa}$ and $P^{\perp}=1-P$. The first component of this decomposition is $\ddot{B}$-acyclic and the second one is $\ddot{d}$ acyclic and killed by $\ddot{B}$. Hence $(\ddot{X}, \ddot{d}, \ddot{b})$ has the Connes property. We finish the section by giving two explicit descriptions of $P(\ddot{X})$ and obtaining a new expression for the connection map of the long exact sequence relating the absolute Hochschild homologies of $A$ and $E$, with the Hochschild homology of $E$ relative to $M$.

Although we had assume that $k$ is a characteristic zero field, many of the results in this paper are valid under considerable weaker hypothesis. More precisely we can take a commutative ring $k$ and a $k$ subalgebra $S$ of $A$ and consider the $S$-relative Hochschild, cyclic, negative and periodic homologies. In this case we must replace $\bar{A}$ by $A / S$ and the tensor products over $k$, that appear in all the complexes in this paper, by cyclic tensor products over $S$ (See $[\mathrm{K} 1, \mathrm{G}-\mathrm{S}], \mathrm{K} 2$ and $\mathrm{Q}$ )). All the results of Section 3 are valid in this context, with the exception of Lemma 3.3 Theorem 3.4 and Propositions 3.5] and 3.8. If $k$ contains $\mathbb{Q}$, then all the results in this paper are valid, except Theorem 4.6] and this theorem is also valid if we also have that $S^{e}$ is semisimple. Finally, when $S^{e}$ is a separable $k$-algebra, the relative and absolute homologies coincide, as was shown in the above mentioned papers.

Next we introduce some notations that we will use throughout this paper.

Notations 1.1. Let $k$ be a commutative ring, $V$ a $k$-module, $C$ a $k$-algebra and $M$ a C-bimodule.

(1) We put $\bar{C}=C / k$ and given $x \in C$ we also let $x$ denote its class in $\bar{C}$.

(2) We let $V^{\otimes n}$ denote the $n$-fold power tensor of $V$.

(3) Given $x_{0} \otimes \cdots \otimes x_{n} \in C \otimes \bar{C}^{\otimes n}$ and $0 \leq i<j \leq n$, we write $\mathbf{x}_{i}^{j}=x_{i} \otimes \cdots \otimes x_{j}$.

(4) For $n \geq 0$, we let $M^{\otimes_{C}^{n}}$ denote the $n$-fold power tensor of $M$ over $C$. As usual, we consider that $M^{\otimes_{C}^{0}}=C$.

(5) Given $x_{1} \otimes_{C} \cdots \otimes_{C} x_{n} \in M^{\otimes_{C}^{n}}$ and $1 \leq i<j \leq n$, we will write $\overline{\mathbf{x}}_{i}^{j}=$ $x_{i} \otimes_{C} \cdots \otimes_{C} x_{j}$. 
Notations 1.2. Let $E=A \ltimes_{f} M$ be a square zero extension.

(1) We let $\pi_{A}: E \rightarrow A$ and $\pi_{M}: E \rightarrow M$ denote the maps defined by $\pi_{A}(a, m)=$ $a$ and $\pi_{M}(a, m)=m$, respectively.

(2) We extend $f$ to $E \otimes E$ writing $f(x, y)=0$ if $x \in M$ or $y \in M$.

(3) Given $x, y \in A \cup M$ we set

$$
x y= \begin{cases}\text { the product of } x \text { and } y \text { in } A & \text { if } x, y \in A, \\ \text { the left action of } x \text { on } y & \text { if } x \in A \text { and } y \in M, \\ \text { the right action of } y \text { on } x & \text { if } x \in M \text { and } y \in A, \\ 0 & \text { if } x, y \in M .\end{cases}
$$

(4) For $0 \leq w \leq n$, let $B_{w}^{n} \subseteq \bar{E}^{\otimes n}$ be the $k$-submodule spanned by the $n$-tensors $x_{1} \otimes \cdots \otimes x_{n}$ such that exactly $w$ of the $x_{i}$ 's belong to $M$, while the other ones belong to $\bar{A}$. To unify expressions we make the convention that $B_{0}^{0}=k$ and $B_{w}^{n}=0$, for $w<0$ or $n<w$.

(5) For $\mathbf{x}_{0}^{n} \in M \otimes B_{w}^{n} \bigcup A \otimes B_{w+1}^{n}$ and $0 \leq l \leq n$, we define $\mu_{l}\left(\mathbf{x}_{0}^{n}\right)$ by

$$
\mu_{l}\left(\mathbf{x}_{0}^{n}\right)= \begin{cases}(-1)^{l} \mathbf{x}_{0}^{l-1} \otimes x_{l} x_{l+1} \otimes \mathbf{x}_{l+2}^{n} & \text { if } 0 \leq l<n, \\ (-1)^{n} x_{n} x_{0} \otimes \mathbf{x}_{1}^{n-1} & \text { If } l=n .\end{cases}
$$

Moreover we set

$$
\begin{array}{ll}
\mu_{0}^{A}\left(\mathbf{x}_{0}^{n}\right)=\pi_{A}\left(x_{0} x_{1}\right) \otimes \mathbf{x}_{2}^{n}, & \mu_{n}^{A}\left(\mathbf{x}_{0}^{n}\right)=(-1)^{n} \pi_{A}\left(x_{n} x_{0}\right) \otimes \mathbf{x}_{1}^{n-1}, \\
\mu_{0}^{M}\left(\mathbf{x}_{0}^{n}\right)=\pi_{M}\left(x_{0} x_{1}\right) \otimes \mathbf{x}_{2}^{n}, & \mu_{n}^{M}\left(\mathbf{x}_{0}^{n}\right)=(-1)^{n} \pi_{M}\left(x_{n} x_{0}\right) \otimes \mathbf{x}_{1}^{n-1} .
\end{array}
$$

(6) For $\mathbf{x}_{0}^{n} \in M \otimes B_{w}^{n} \bigcup A \otimes B_{w+1}^{n}$ and $0 \leq j \leq n$, we define $\mu_{l}\left(\mathbf{x}_{0}^{n}\right)$ by

$$
F_{j}\left(\mathbf{x}_{0}^{n}\right)= \begin{cases}(-1)^{j} \mathbf{x}_{0}^{j-1} \otimes f\left(x_{j}, x_{j+1}\right) \otimes \mathbf{x}_{j+2}^{n} & \text { if } 0 \leq j<n, \\ -\mathbf{x}_{1}^{n-1} \otimes f\left(x_{n}, x_{0}\right) & \text { If } j=n .\end{cases}
$$

(7) For an elementary tensor $\mathbf{x}_{0}^{n}$, such that $x_{i} \in A \bigcup M$ for all $i$, we let $i\left(\mathbf{x}_{0}^{n}\right)$ denote the last index $i$ such that $x_{i} \in M$.

(8) For an elementary tensor $\mathbf{x}_{0}^{n}$ such that $x_{i} \in A \bigcup M$ for all $i$, we define $t\left(\mathbf{x}_{0}^{n}\right)=(-1)^{i\left(\mathbf{x}_{0}^{n}\right) n} \mathbf{x}_{i\left(\mathbf{x}_{0}^{n}\right)}^{n} \otimes \mathbf{x}_{0}^{i\left(\mathbf{x}_{0}^{n}\right)-1}$.

Acknowledgement. We would like to thank our colleague, Professor Guillermo Cortiñas for his careful reading of a first version of our paper and for his suggestions that have helped us to improve the presentation of this paper substantially.

\section{Preliminaries}

In this section we recall some well known definitions and results, and we fix some notations that we will use in the rest of the paper. Let $C$ be a $k$-algebra.

2.1. Double and triple complexes. A double complex $\mathcal{X}=\left(X, d^{v}, d^{h}\right)$ of $C$ modules, is a family $\left(X_{p q}\right)_{p, q \in \mathbb{Z}}$ of $C$-modules, together with $C$-linear maps

$$
d^{h}: X_{p q} \rightarrow X_{p-1, q} \text { and } d^{v}: X_{p q} \rightarrow X_{p, q-1}
$$

such that $d^{h} \circ d^{h}=0, d^{v} \circ d^{v}=0$ and $d^{v} \circ d^{h}+d^{h} \circ d^{v}=0$. The total complex of $\left(X, d^{v}, d^{h}\right)$ is the complex $\operatorname{Tot}(\mathcal{X})=(X, d)$, in which

$$
X_{n}=\prod_{p} X_{p, n-p} \quad \text { and } \quad d=d^{v}+d^{h} .
$$


A morphism of double complexes $f:\left(X, d^{v}, d^{h}\right) \rightarrow\left(Y, \delta^{v}, \delta^{h}\right)$ is a family of maps $f: X_{p q} \rightarrow Y_{p q}$, such that $\delta^{v} \circ f=f \circ d^{v}$ and $\delta^{h} \circ f=f \circ d^{h}$. The morphism from $\operatorname{Tot}\left(X, d^{v}, d^{h}\right)$ to $\operatorname{Tot}\left(Y, \delta^{v}, \delta^{h}\right)$ induced by $f$ will be denoted $\operatorname{Tot}(f)$.

Similarly, one can give the notions of triple complex $\mathcal{X}=\left(X, d^{v}, d^{h}, d^{d}\right)$ and of morphism of triple complexes. For a triple complex $\mathcal{X}$, there are three ways for constructing a double complex by taking total complexes of double complexes. We call each one of these double complexes a partial total complex of $\mathcal{X}$. Finally, the total complex $\operatorname{Tot}(\mathcal{X})$ of $\mathcal{X}$, is the total complex of any of its partial total complexes. Of course, $\operatorname{Tot}(\mathcal{X})$ is independently of the chosen way to construct it.

2.2. Mixed complexes. In this subsection we recall briefly the notion of mixed complex. For more details about this concept we refer to Ka1 and $\mathrm{B}$.

A mixed complex $(X, b, B)$ is a graded $C$-module $\left(X_{n}\right)_{n \geq 0}$, endowed with morphisms $b: X_{n} \rightarrow X_{n-1}$ and $B: X_{n} \rightarrow X_{n+1}$, such that

$$
b \circ b=0, \quad B \circ B=0 \quad \text { and } \quad B \circ b+b \circ B=0 .
$$

A morphism of mixed complexes $f:(X, b, B) \rightarrow(Y, d, D)$ is a family $f: X_{n} \rightarrow Y_{n}$, such that $d \circ f=f \circ b$ and $D \circ f=f \circ B$. A mixed complex $\mathcal{X}=(X, b, B)$ determines a double complex

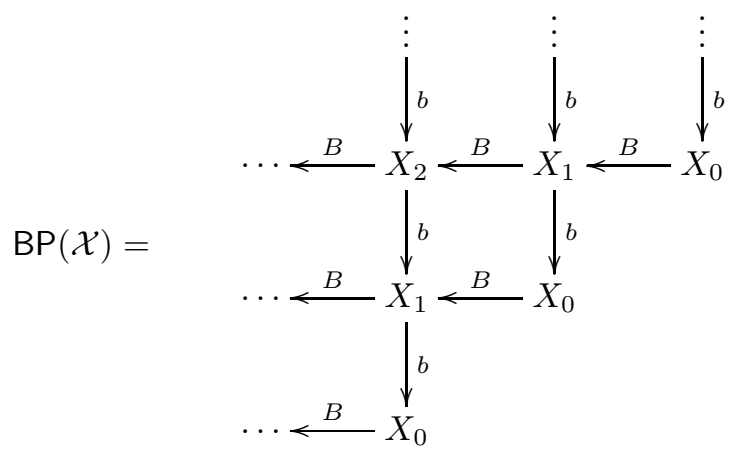

By deleting the positively numbered columns we obtain a subcomplex $\operatorname{BN}(\mathcal{X})$ of $\mathrm{BP}(\mathcal{X})$. The quotient double complex $\mathrm{BP}(\mathcal{X}) / \mathrm{BN}(\mathcal{X})$ is denoted by $\mathrm{BC}(\mathcal{X})$. The homologies $\mathrm{HC}_{*}(\mathcal{X}), \mathrm{HN}_{*}(\mathcal{X})$ and $\mathrm{HP}_{*}(\mathcal{X})$, of the total complexes of $\mathrm{BC}(\mathcal{X}), \mathrm{BN}(\mathcal{X})$ and $\mathrm{BP}(\mathcal{X})$ respectively, are called the cyclic, negative and periodic homologies of $\mathcal{X}$. The homology $\mathrm{HH}_{*}(\mathcal{X})$, of $(X, b)$, is called the Hochschild homology of $\mathcal{X}$. Finally, it is clear that a morphism $f: \mathcal{X} \rightarrow \mathcal{Y}$ of mixed complexes induces a morphism from the double complex $\operatorname{BP}(\mathcal{X})$ to the double complex $\operatorname{BP}(\mathcal{Y})$.

Following $[\mathrm{Co}$ by a double mixed complex we will understand a bigraded module $X$ equipped with three $k$-linear maps of degree \pm 1 : $\partial$ that lowers the first index and fixes the second one, $\delta$ that fixes the first index and lowers the second one, and $B$ which fixes the first index and increases the second one. These maps satisfy

$$
0=\partial^{2}=\delta^{2}=B^{2}=\delta \circ \partial+\partial \circ \delta=\delta \circ B+B \circ \delta=\partial \circ B+B \circ \partial .
$$

The mixed complex $(X, \delta+\partial, B)$ associated with a double mixed complex $(X, \delta, \partial, B)$ is obtained setting $(X, \delta+\partial)=\operatorname{Tot}(X, \delta, \partial)$ and $B_{n}=\bigoplus_{i+j=n} B_{i j}$. By definition, the Hochschild, cyclic, periodic and negative homologies of $(X, \delta, \partial, B)$ are the Hochschild, cyclic, periodic and negative homologies of $(X, \delta+\partial, B)$, respectively.

2.3. The relative Hochschild and cyclic homologies. Let $C$ be a $k$-algebra and let $\left(C \otimes \bar{C}^{\otimes *}, b, B\right)$ be the normalized mixed complex of $C$. Recall that the cyclic, negative, periodic and Hochschild homologies $\mathrm{HC}_{*}(C), \mathrm{HN}_{*}(C), \mathrm{HP}_{*}(C)$ and $\mathrm{HH}_{*}(C)$ of $C$ are the respective homologies of $\left(C \otimes \bar{C}^{\otimes *}, b, B\right)$. 
Next, we define the relative homologies. Let $I$ be a two sided ideal of $C$ and let $D=C / I$. The cyclic, negative, periodic and Hochschild homologies $\mathrm{HC}_{*}(C, I)$, $\mathrm{HN}_{*}(C, I), \mathrm{HP}_{*}(C, I)$ and $\mathrm{HH}_{*}(C, I)$, of $C$ relative to $I$, are by definition the respective homologies of the mixed complex

$$
\operatorname{ker}\left(\left(C \otimes \bar{C}^{\otimes *}, b, B\right) \stackrel{\pi}{\longrightarrow}\left(D \otimes \bar{D}^{\otimes *}, b, B\right)\right),
$$

where $\pi$ is the map induced by the canonical projection from $C$ onto $D$.

2.4. The perturbation lemma. Next, we recall the perturbation lemma. We give the more general version introduced in [C].

A homotopy equivalence data

$$
(Y, \partial) \underset{i}{\stackrel{p}{\rightleftarrows}}(X, d), \quad h: X_{*} \rightarrow X_{*+1},
$$

consists of the following:

(1) Chain complexes $(Y, \partial),(X, d)$ and quasi-isomorphisms $i, p$ between them,

(2) A homotopy $h$ from $i \circ p$ to id.

A perturbation $\delta$ of (2) is a map $\delta: X_{*} \rightarrow X_{*-1}$ such that $(d+\delta)^{2}=0$. We call it small if id $-\delta \circ h$ is invertible. In this case we write $A=(\text { id }-\delta \circ h)^{-1} \circ \delta$ and we consider

$$
\left(Y, \partial^{1}\right) \underset{i^{1}}{\stackrel{p^{1}}{\leftrightarrows}}(X, d+\delta), \quad h^{1}: X_{*} \rightarrow X_{*+1},
$$

with

$$
\partial^{1}=\partial+p \circ A \circ i, \quad i^{1}=i+h \circ A \circ i, \quad p^{1}=p+p \circ A \circ h, \quad h^{1}=h+h \circ A \circ h .
$$

A deformation retract is a homotopy equivalence data such that $p \circ i=i d$. A deformation retract is called special if $h \circ i=0, p \circ h=0$ and $h \circ h=0$.

In all the cases considered in this paper the map $\delta \circ h$ is locally nilpotent, and so $(\text { id }-\delta \circ h)^{-1}=\sum_{n=0}^{\infty}(\delta \circ h)^{n}$.

Theorem 2.1. ([C]) If $\delta$ is a small perturbation of the homotopy equivalence data (2), then the perturbed data (3) is a homotopy equivalence. Moreover, if (2) is a special deformation retract, then (3) is also.

2.5. The suspension. The suspension of a chain complex $(X, d)$ is the complex $(X, d)[1]=(X[1], d[1])$, defined by $X[1]_{*}=X_{*-1}$ and $d[1]_{*}=-d_{*-1}$.

\section{The Relative CyClic homology of a SQUARE ZERO EXTENSION}

Let $A$ be a $k$-algebra, $M$ an $A$-bimodule and $f: A \otimes A \rightarrow M$ a Hochschild normal 2-cocycle. The square zero extension $E=A \ltimes_{f} M$, of $A$ by $M$ associated with $f$, is the direct sum $A \oplus M$ with the associative algebra structure given by

$$
(a, m)\left(a^{\prime}, m^{\prime}\right)=\left(a a^{\prime}, a m^{\prime}+m a^{\prime}+f\left(a, a^{\prime}\right)\right) .
$$

Let $C$ be a $k$-algebra and let $M$ be a two sided ideal of $C$ such that $M^{2}=0$. It is well known that $C$ is isomorphic to a square zero extension $E$ of $A$ by $M$. In this section we obtain a double mixed complex, simpler than the canonical one, giving the Hochschild, cyclic, periodic and negative homologies of $E$ relative to $M$. Then we show that the cyclic homology of $E$ relative to $M$, is also given by a still simpler complex. Finally, we obtain convenient expressions for the connection map of the 
long exact sequences in Hochschild and cyclic homologies, associated with the short exact sequence of mixed complexes

$$
0 \longrightarrow \operatorname{ker}(\pi) \stackrel{i}{\longrightarrow}\left(E \otimes \bar{E}^{\otimes *}, b, B\right) \stackrel{\pi}{\longrightarrow}\left(A \otimes \bar{A}^{\otimes *}, b, B\right) \longrightarrow 0 .
$$

3.1. Complexes for the relative Hochschild, cyclic, periodic and negative homologies. For $w \geq 0$ and $v \geq 2 w$, let $X_{v}^{w}=M \otimes B_{w}^{v-w}$. By convenience we put $X_{v}^{w}=0$, otherwise. From now on we often will use the indices $v, w$ and $n$, which always will satisfy the relation $n=v-w$. Consider the triple diagram

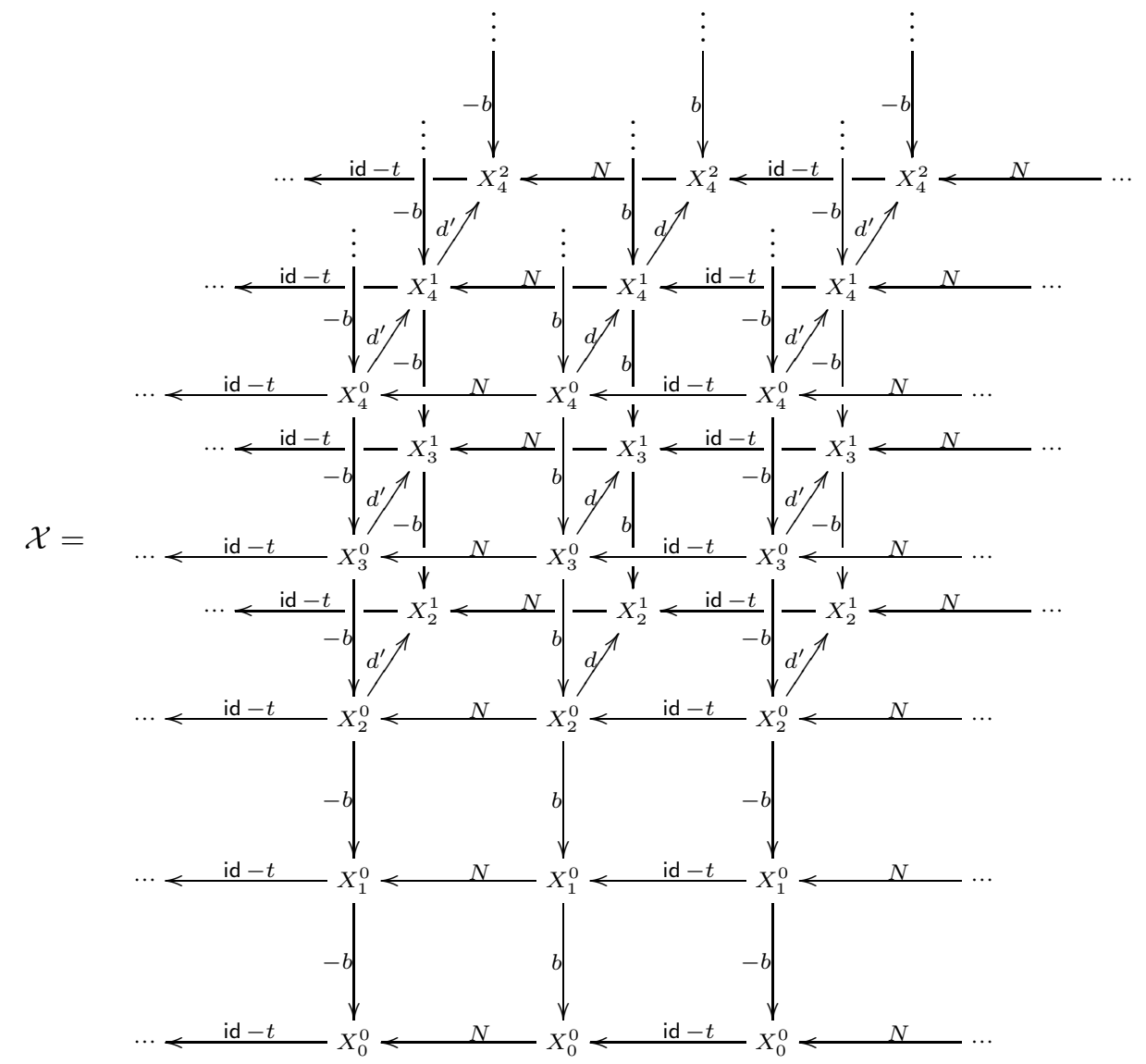

where

$$
\begin{array}{ll}
b\left(\mathbf{x}_{0}^{n}\right)=\sum_{j=0}^{n} \mu_{j}\left(\mathbf{x}_{0}^{n}\right), & d\left(\mathbf{x}_{0}^{n}\right)=\sum_{j=1}^{n-1} F_{j}\left(\mathbf{x}_{0}^{n}\right), \\
d^{\prime}\left(\mathbf{x}_{0}^{n}\right)=-d\left(\mathbf{x}_{0}^{n}\right)-\sum_{j=i\left(\mathbf{x}_{0}^{n}\right)+1}^{n-1} t\left(F_{j}\left(\mathbf{x}_{0}^{n}\right)\right), & N\left(\mathbf{x}_{0}^{n}\right)=\sum_{l=0}^{w} t^{l}\left(\mathbf{x}_{0}^{n}\right),
\end{array}
$$

the middle face $(X, b, d)$ is the 0 -th face and the bottom row is the 0 -th row. Note that id $-t: X_{v}^{0} \rightarrow X_{v}^{0}$ is the zero map and $N: X_{v}^{0} \rightarrow X_{v}^{0}$ is the identity map.

For $l \in \mathbb{Z}$, let $\tau^{l}(\mathcal{X})$ be the subdiagram of $\mathcal{X}$ obtaining by deleting the $u$-th faces $(X, b, d)$ and $\left(X,-b, d^{\prime}\right)$ with $u>l$, and let $\tau_{0}(\mathcal{X})$ and $\tau_{0}^{1}(\mathcal{X})$ be the quotient triple diagrams of $\mathcal{X}$ by $\tau^{-1}(\mathcal{X})$ and $\tau^{1}(\mathcal{X})$ by $\tau^{-1}(\mathcal{X})$, respectively. 
Theorem 3.1. $\mathcal{X}$ is a triple complex. Moreover

$$
\begin{aligned}
& \mathrm{HH}_{*}(E, M)=\mathrm{H}_{*}\left(\operatorname{Tot}\left(\tau_{0}^{1}(\mathcal{X})\right)\right), \\
& \mathrm{HC}_{*}(E, M)=\mathrm{H}_{*}\left(\operatorname{Tot}\left(\tau_{0}(\mathcal{X})\right)\right), \\
& \mathrm{HP}_{*}(E, M)=\mathrm{H}_{*}(\operatorname{Tot}(\mathcal{X})), \\
& \operatorname{HN}_{*}(E, M)=\mathrm{H}_{*}\left(\operatorname{Tot}\left(\tau^{1}(\mathcal{X})\right)\right) .
\end{aligned}
$$

Consequently, the following equalities hold:

$$
\begin{array}{lll}
d \circ b=-b \circ d, & d^{\prime} \circ b=-b \circ d^{\prime}, & d^{\prime} \circ N=-N \circ d, \\
d \circ(\text { id }-t)=-(\text { id }-t) \circ d^{\prime}, & b \circ N=N \circ b, & t \circ b=b \circ t .
\end{array}
$$

We will use these equalities freely throughout the paper.

Theorem 3.1 is a consequence of Theorem 3.2 which we enounce below and whose proof will be relegated to Appendix A. For $w \geq 0$ and $v \geq 2 w$, let $\hat{X}_{v}^{w}=X_{v}^{w} \oplus X_{v-1}^{w}$. Consider the diagram $(\hat{X}, \hat{b}, \hat{d})$ where the maps $\hat{b}: \hat{X}_{v}^{w} \rightarrow \hat{X}_{v-1}^{w}$ and $\hat{d}: \hat{X}_{v}^{w} \rightarrow \hat{X}_{v}^{w+1}$ are defined by

$$
\hat{b}(\mathbf{x}, \mathbf{y})=(b(\mathbf{x})+(\text { id }-t)(\mathbf{y}),-b(\mathbf{y})) \quad \text { and } \quad \hat{d}(\mathbf{x}, \mathbf{y})=\left(d(\mathbf{x}), d^{\prime}(\mathbf{y})\right) .
$$

Note that $(\hat{X}, \hat{b}, \hat{d})$ is one of the partial total complexes of the triple complex $\tau_{0}^{1}(\mathcal{X})$, and so $\operatorname{Tot}\left(\tau_{0}^{1}(\mathcal{X})\right)=\operatorname{Tot}(\hat{X}, \hat{b}, \hat{d})$ (but we have not proved that $\tau_{0}^{1}(\mathcal{X})$ is a triple complex, yet). Let $\hat{B}: \hat{X}_{v}^{w} \rightarrow \hat{X}_{v+1}^{w}$ be the map defined by $\hat{B}(\mathbf{x}, \mathbf{y})=(0, N(\mathbf{x}))$.

Theorem 3.2. The following assertions hold:

(1) $(\hat{X}, \hat{b}, \hat{d}, \hat{B})$ is a double mixed complex.

(2) The Hochschild, cyclic, periodic and negative homologies of $(\hat{X}, \hat{b}, \hat{d}, \hat{B})$ are the Hochschild, cyclic, periodic and negative homologies of $E$ relative to $M$, respectively.

Proof. See Appendix A.

Proof of Theorem 3.1 Let $(\breve{X}, \breve{b}, \breve{B})$ be the mixed complex associated with $(\hat{X}, \hat{b}, \hat{d}, \hat{B})$. Theorem 3.1 follows immediately from Theorem 3.2 and the fact that

$$
\begin{aligned}
& \operatorname{Tot}\left(\tau_{0}^{1}(\mathcal{X})\right)=(\breve{X}, \breve{b}), \\
& \operatorname{Tot}\left(\tau_{0}(\mathcal{X})\right)=\operatorname{Tot}(\operatorname{BC}(\breve{X}, \breve{b}, \breve{B})), \\
& \operatorname{Tot}(\mathcal{X})=\operatorname{Tot}(\operatorname{BP}(\breve{X}, \breve{b}, \breve{B})), \\
& \operatorname{Tot}\left(\tau^{1}(\mathcal{X})\right)=\operatorname{Tot}(\operatorname{BN}(\breve{X}, \breve{b}, \breve{B})),
\end{aligned}
$$

which can be easily checked.

Lemma 3.3. The rows of the triple complex $\mathcal{X}$ are contractible.

Proof. For $w \geq 0$ and $v \geq 2 w$, let $\sigma, \sigma^{\prime}: X_{v}^{w} \rightarrow X_{v}^{w}$ be the maps defined by $\sigma=\frac{1}{w+1}$ id and $\sigma^{\prime}=\sum_{j=0}^{w-1} \frac{w-j}{w+1} t^{j}$. A direct computation shows that:

$$
\begin{aligned}
& \sigma \circ N=N \circ \sigma=\frac{1}{w+1} N, \\
& (\mathrm{id}-t) \circ \sigma^{\prime}=\sigma^{\prime} \circ(\mathrm{id}-t)=\sum_{j=0}^{w-1} \frac{w-j}{w+1} t^{j}-\sum_{j=1}^{w} \frac{w-j+1}{w+1} t^{j}=\mathrm{id}-\frac{1}{w+1} N .
\end{aligned}
$$

The result follows immediately from these equalities. 
Theorem 3.4. Let $(\bar{X}, \bar{b}, \bar{d})$ be the cokernel of id $-t:\left(X,-b, d^{\prime}\right) \rightarrow(X, b, d)$. The relative cyclic homology $\mathrm{HC}_{*}(E, M)$ is the homology of $(\bar{X}, \bar{b}, \bar{d})$.

Proof. This follows immediately from Theorem 3.1 and Lemma 3.3.

From Lemma 3.3 it follows also that $(\hat{X}, \hat{b}, \hat{d}, \hat{B})$ has the Connes property. Theorem 3.4 can be alternatively deduced from this fact.

Let $C$ be a $k$-algebra, $M$ a two sided ideal of $C$ and $A=C / M$. Next we give an alternative proof of a celebrated theorem of Goodwillie obtained in G2. To do this we need the following result:

Proposition 3.5. It is true that $b \circ \sigma^{\prime}=\sigma^{\prime} \circ b$.

Proof. Fix $w \geq 0$ and $v>2 w$ and let $\mathbf{x}_{0}^{n} \in X_{v}^{w}$ be an elementary tensor. Let $0=i_{0}<i_{1}<\cdots<i_{w} \leq n$ be the indexes such that $x_{i_{j}} \in M$ and set $i_{w+1}=n+1$. A direct computation shows that

$$
\mu_{l} \circ t^{j}\left(\mathbf{x}_{0}^{n}\right)= \begin{cases}t^{j} \circ \mu_{l+i_{w+1-j}}\left(\mathbf{x}_{0}^{n}\right) & \text { if } 0 \leq l \leq n-i_{w+1-j}, \\ t^{j} \circ \mu_{l-n-1+i_{w+1-j}}\left(\mathbf{x}_{0}^{n}\right) & \text { if } n-i_{w+1-j}<l \leq n .\end{cases}
$$

Hence, the families $\mu_{l} \circ t^{j}\left(\mathbf{x}_{0}^{n}\right)$ and $t^{j} \circ \mu_{l}\left(\mathbf{x}_{0}^{n}\right)$ coincide, and so

$$
b \circ \sigma^{\prime}\left(\mathbf{x}_{0}^{n}\right)=\sum_{l=0}^{n} \sum_{j=0}^{w-1} \frac{w-j}{w+1} \mu_{l} \circ t^{j}\left(\mathbf{x}_{0}^{n}\right)=\sum_{j=0}^{w-1} \sum_{l=0}^{n} \frac{w-j}{w+1} t^{j} \circ \mu_{l}\left(\mathbf{x}_{0}^{n}\right)=\sigma^{\prime} \circ b\left(\mathbf{x}_{0}^{n}\right),
$$

as we want.

Theorem 3.6. (Goodwillie) Let $C$ be a k-algebra, $M$ a two-sided ideal of $C$ and $A=C / M$. If $M$ is nilpotent, then $\operatorname{HP}_{*}(C)=\operatorname{HP}_{*}(A)$.

Proof. Without loss of generality we can assume that $M^{2}=0$. So $C$ is isomorphic to a squared zero extension $E=A \ltimes_{f} M$. We prove the theorem showing that $\mathrm{HP}_{*}(E, M)=0$. Let us consider the filtration $\left(F_{q}(\mathcal{X})\right)_{q \geq 0}$ of $\mathcal{X}$, given by $F_{q}\left(X_{v}^{w}\right)=$ $X_{v}^{w}$ if $w \geq q$ and $F_{q}\left(X_{v}^{w}\right)=0$ otherwise. Taking the graded complex associated with this filtration we can assume that $E$ the zero squared extension $E=A \ltimes M$, with trivial cocycle. In this case $\mathcal{X}$ is contractible, since $d=d^{\prime}=0$ and, by Proposition 3.5, $b \circ \sigma^{\prime}=\sigma^{\prime} \circ$.

\subsection{The connection map for the Hochschild homology. Let}

$$
\cdots \rightarrow \mathrm{HH}_{n}(E, M) \rightarrow \mathrm{HH}_{n}(E) \rightarrow \mathrm{HH}_{n}(A) \stackrel{\delta_{n}}{\rightarrow} \mathrm{HH}_{n-1}(E, M) \rightarrow \mathrm{HH}_{n-1}(E) \rightarrow \cdots
$$

be the long exact sequence associated with the short exact sequence (4). In this subsection we obtain a morphism of complexes $\breve{\delta}:\left(A \otimes \bar{A}^{\otimes *}, b\right) \rightarrow(\breve{X}, \breve{b})[1]$, inducing the maps $\delta_{n}$. For each $n \geq 0$, let

$$
\delta^{1}: A \otimes \bar{A}^{\otimes n} \rightarrow X_{n-1}^{0}, \quad \delta^{2}: A \otimes \bar{A}^{\otimes n} \rightarrow X_{n-2}^{0} \quad \text { and } \quad \delta^{3}: A \otimes \bar{A}^{\otimes n} \rightarrow X_{n-1}^{1}
$$

be the maps defined by

$$
\delta^{1}=\sum_{j=0}^{n} t_{\circ} F_{j}, \quad \delta^{2}=\mu_{0} \circ F_{1} \quad \text { and } \quad \delta^{3}=\sum_{i=2}^{n-1} F_{0} \circ F_{i}-\sum_{0 \leq i<j \leq n} t_{\circ} F_{i} \circ F_{j} .
$$

The proof of Proposition 3.7 below and Proposition 3.8 in the next subsection are relegated to Appendix A.

Proposition 3.7. The connection map $\delta_{n}: \mathrm{HH}_{n}(A) \rightarrow \mathrm{HH}_{n-1}(E, M)$ is induced by the morphism of complexes $\breve{\delta}:\left(A \otimes \bar{A}^{\otimes *}, b\right) \rightarrow(\breve{X}, \breve{b})[1]$, given by

$$
\breve{\delta}(\mathbf{a})=\left(\delta^{2}(\mathbf{a}), \delta^{1}(\mathbf{a}), \delta^{3}(\mathbf{a}), 0,0, \ldots\right),
$$


where we are writing

$$
\breve{X}_{n-1}=X_{n-2}^{0} \oplus X_{n-1}^{0} \oplus X_{n-1}^{1} \oplus X_{n}^{1} \oplus X_{n}^{2} \oplus X_{n+1}^{2} \oplus X_{n+1}^{3} \oplus X_{n+2}^{3} \oplus \cdots
$$

Proof. See Appendix A.

\subsection{The connection map for the cyclic homology. Let}

$$
\cdots \rightarrow \mathrm{HC}_{n}(E, M) \rightarrow \mathrm{HC}_{n}(E) \rightarrow \mathrm{HC}_{n}(A) \stackrel{\delta_{n}}{\rightarrow} \mathrm{HC}_{n-1}(E, M) \rightarrow \mathrm{HC}_{n-1}(E) \rightarrow \cdots
$$

be the long exact sequence associated with the short exact sequence (4).

Proposition 3.8. The connection map $\delta_{n}: \mathrm{HC}_{n}(A) \rightarrow \mathrm{HC}_{n-1}(E, M)$ is induced by the morphism of complexes $\bar{\delta}: \operatorname{Tot}\left(\mathrm{BC}\left(A \otimes \bar{A}^{\otimes *}, b, B\right)\right) \rightarrow \operatorname{Tot}(\bar{X}, \bar{b}, \bar{d})[1]$, given by $\bar{\delta}\left(\mathbf{a}_{0}^{n}, \mathbf{b}_{0}^{n-2}, \mathbf{c}_{0}^{n-4}, \ldots\right)=\delta^{1}\left(\mathbf{a}_{0}^{n}\right)$. Note that the image of $\bar{\delta}$ is included in $\bar{X}_{n-1}^{0}$.

Proof. See Appendix A.

\section{The HARMONIC DECOMPOSITION}

As in the proof of Theorem 3.1 we let $(\breve{X}, \breve{b}, \breve{B})$ denote the mixed complex associated with the double mixed complex $(\hat{X}, \hat{b}, \hat{d}, \hat{B})$, introduced in Theorem 3.2 The aim of this section is to show that $(\breve{X}, \breve{b}, \breve{B})$ has a harmonic decomposition like the one studied in $\mathrm{C}-\mathrm{Q}$. In order to carry out this task we need to define a de Rham coboundary map and a Karoubi operator on $(\breve{X}, \breve{b})$. As we said in the introduction we are going to work with a new double mixed complex $(\ddot{X}, \ddot{d}, \ddot{b}, \ddot{B})$, whose associated mixed complex is also $(\breve{X}, \breve{b}, \breve{B})$. In the first three subsections we follow closely the exposition of $\mathrm{C}-\mathrm{Q}$.

4.1. The Rham coboundary map and the Karoubi operator. It is easy to see that $\tau_{0}^{1}(\mathcal{X})$ is the total complex of the double complex

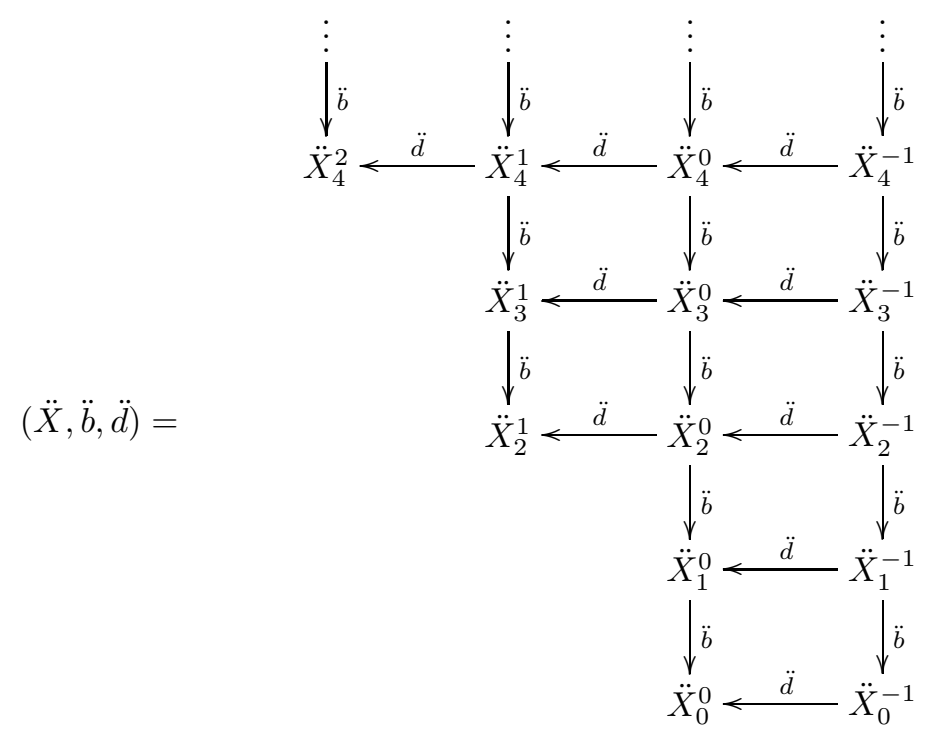

where $\ddot{X}_{v}^{w}=X_{v}^{w} \oplus X_{v}^{w+1}$ and the boundary maps are defined by

$$
\ddot{b}(\mathbf{x}, \mathbf{y})=(b(\mathbf{x}),-b(\mathbf{y})) \quad \text { and } \quad \ddot{d}(\mathbf{x}, \mathbf{y})=\left(d(\mathbf{x})+(\mathrm{id}-t)(\mathbf{y}), d^{\prime}(\mathbf{y})\right) .
$$


The de Rham coboundary map $\ddot{d}_{R}: \ddot{X}_{v}^{w} \rightarrow \ddot{X}_{v}^{w-1}$ is defined by $\ddot{d}_{R}(\mathbf{x}, \mathbf{y})=(0, \mathbf{x})$. It is immediate that $(\ddot{X}, \ddot{d} R)$ is acyclic. We now define the Karoubi operator of $\ddot{X}$. Let $\ddot{\kappa}(0): \ddot{X}_{v}^{w} \rightarrow \ddot{X}_{v}^{w}$ and $\ddot{\kappa}(1): \ddot{X}_{v}^{w} \rightarrow \ddot{X}_{v}^{w}$ be the maps defined by

$$
\ddot{\kappa}(0)(\mathbf{x}, \mathbf{y})=(t(\mathbf{x}), t(\mathbf{y})) \text { and } \ddot{\kappa}(1)(\mathbf{x}, \mathbf{y})=\left(0, d(\mathbf{x})-d^{\prime}(\mathbf{x})\right) .
$$

The Karoubi operator $\ddot{\kappa}$ of $\ddot{X}$ is the degree zero operator defined by

$$
\ddot{\kappa}=\ddot{\kappa}(0)+\ddot{\kappa}(1) .
$$

Let $\breve{d} R: \breve{X}_{n} \rightarrow \breve{X}_{n+1}$ and $\breve{\kappa}: \breve{X}_{n} \rightarrow \breve{X}_{n}$ be the maps defined by

$$
\breve{d} R_{n}=\bigoplus_{w=-1}^{n} \ddot{d}_{R_{n+w}}^{w} \quad \text { and } \quad \breve{\kappa}_{n}=\bigoplus_{w=-1}^{n} \ddot{\kappa}_{n+w}^{w},
$$

respectively. A direct computation shows that

$$
\mathrm{id}-\ddot{\kappa}=\ddot{d} \circ \ddot{d}_{R}+\ddot{d}_{R} \circ \ddot{d} \quad \text { and } \quad 0=\ddot{b} \circ \ddot{d}_{R}+\ddot{d}_{R} \circ \ddot{b} .
$$

In particular, $\ddot{\kappa}$ is homotopic to the identity with respect to either of the differentials $\ddot{d}, \ddot{d}_{R}$, and so it commutes with them. From (5) it follows that

$$
\text { id }-\breve{\kappa}=\breve{b} \circ \breve{d} R+\breve{d} R \circ \breve{b} \text {. }
$$

Consequently, $\breve{\kappa}$ commutes with $\breve{b}$ and $\breve{d} R$. Hence, $\ddot{\kappa}$ also commutes with $\ddot{b}$ (which can be also proved by a direct computation). Let $\ddot{B}: \ddot{X}_{v}^{w} \rightarrow \ddot{X}_{v}^{w-1}$ be the map defined by $\ddot{B}(\mathbf{x}, \mathbf{y})=(0, N(\mathbf{x}))$. An easy computation shows that $(\ddot{X}, \ddot{d}, \ddot{b}, \ddot{B})$ is a double mixed complex and that its associated mixed complex is $(\breve{X}, \breve{b}, \breve{B})$. Furthermore, $\ddot{B}(\mathbf{x})=\sum_{i=0}^{w} \ddot{\kappa}^{i} \ddot{d}_{R}(\mathbf{x})$ for all $\mathbf{x} \in \ddot{X}_{v}^{w}$. Using this we obtain:

$$
\ddot{B} \circ \ddot{\kappa}=\ddot{\kappa} \circ \ddot{B}=\ddot{B} \quad \text { and } \quad \ddot{d}_{R} \circ \ddot{B}=\ddot{B} \circ \ddot{d}_{R}=0 .
$$

4.2. The harmonic decomposition. From the definition of $\ddot{\kappa}$ it follows immediately that

$$
\left(\ddot{\kappa}^{w+2}-\mathrm{id}\right) \circ\left(\ddot{\kappa}^{w+1}-\mathrm{id}\right)\left(\ddot{X}_{v}^{w}\right) \subseteq\left(\kappa^{w+2}-\mathrm{id}\right)\left(X_{1 v}^{w+1}\right)=0 .
$$

This implies that $\ddot{\kappa}$ satisfies the polynomial equation $P_{w}(\ddot{\kappa})=0$ on $\ddot{X}_{v}^{w}$, where

$$
P_{w}=\left(X^{w+1}-1\right)\left(X^{w+2}-1\right) .
$$

The roots of $P_{w}$ are the $r$-th roots of unity, with $r=w+1$ and $r=w+2$. Moreover, 1 is a double root and the all other roots are simple. Consequently $\ddot{X}_{v}^{w}$ decomposes into the direct sum of the generalized eigenspace $\operatorname{ker}(\ddot{\kappa}-\text { id })^{2}$ and its complement $\operatorname{Im}(\ddot{\kappa}-\mathrm{id})^{2}$. Combining this for all $v, w$ we obtain the following decomposition

$$
\ddot{X}=\operatorname{ker}(\ddot{\kappa}-\mathrm{id})^{2} \oplus \operatorname{Im}(\ddot{\kappa}-\mathrm{id})^{2},
$$

Each of these generalized subspaces is stable under any operator commuting with $\ddot{\kappa}$, for instance, $\ddot{b}, \ddot{d}, \ddot{d} R$ and $\ddot{B}$.

4.3. The harmonic projection and the Green operator. Let $P$ be the harmonic projection operator, which is the identity map on $\operatorname{ker}(\ddot{\kappa}-\mathrm{id})^{2}$ and the zero map on $\operatorname{Im}(\ddot{\kappa}-\mathrm{id})^{2}$. Thus we have

$$
\ddot{X}=P(\ddot{X}) \oplus P^{\perp}(\ddot{X}),
$$

where $P^{\perp}=$ id $-P$. It is immediate that $(P(\ddot{X}), \ddot{d}, \ddot{b}, \ddot{B})$ and $\left(P^{\perp}(\ddot{X}), \ddot{d}, \ddot{b}, \ddot{B}\right)$ are double mixed subcomplexes of $(\ddot{X}, \ddot{d}, \ddot{b}, \ddot{B})$. On $P^{\perp}(\ddot{X})$ the operator

$$
\text { id }-\ddot{\kappa}=\ddot{d} \circ \ddot{d}_{R}+\ddot{d}_{R} \circ \ddot{d}
$$


is both invertible and homotopic to zero with respect to either differential $\ddot{d}$ and $\ddot{d}_{R}$. Hence the complexes $\left(P^{\perp}(\ddot{X}), \ddot{d}\right)$ and $\left(P^{\perp}(\ddot{X}), \ddot{d}_{R}\right)$ are acyclic. Let

$$
P\left(\breve{X}_{n}\right)=\bigoplus_{w=-1}^{n} P\left(\ddot{X}_{n+w}^{w}\right) \quad \text { and } \quad P^{\perp}\left(\breve{X}_{n}\right)=\bigoplus_{w=-1}^{n} P^{\perp}\left(\ddot{X}_{n+w}^{w}\right) .
$$

The same argument shows that $\left(P^{\perp}(\breve{X}), \breve{b}\right)$ and $\left(P^{\perp}(\breve{X}), \breve{d} R\right)$ are also acyclic. The Green operator $G: \ddot{X} \rightarrow \ddot{X}$ is defined to be zero on $P(\ddot{X})$ and the inverse of id $-\ddot{\kappa}$ on $P^{\perp}(\ddot{X})$. It is immediate that

$$
G \circ P=P \circ G=0 \quad \text { and } \quad P^{\perp}=G \circ(\text { id }-\ddot{\kappa})=G \circ\left(\ddot{d}_{\circ} \ddot{d}_{R}+\ddot{d}_{R^{\circ}} \ddot{d}\right) .
$$

Moreover $P$ and $G$ commute with each operator that commutes with $\ddot{\kappa}$.

Proposition 4.1. One has

$$
P^{\perp}(\ddot{X})=\ddot{d} \circ P^{\perp}(\ddot{X}) \oplus \ddot{d}_{R} \circ P^{\perp}(\ddot{X}) .
$$

Furthermore $\ddot{d}_{R}$ maps $\ddot{d_{\circ}} P^{\perp}(\ddot{X})$ isomorphically onto $\ddot{d}_{R^{\circ}} P^{\perp}(\ddot{X})$ with inverse $G \circ \ddot{d}$ and $\ddot{d}$ maps $\ddot{d} R^{\circ} P^{\perp}(\ddot{X})$ isomorphically onto $\ddot{{ }^{\circ}} P^{\perp}(\ddot{X})$ with inverse $G^{\circ} \ddot{d}_{R}$. This gives a new proof that $\left(P^{\perp}(\ddot{X}), \ddot{d}\right)$ and $\left(P^{\perp}(\ddot{X}), \ddot{d}_{R}\right)$ are acyclic.

Proof. The proof of Proposition 2.1 of $[\mathrm{C}-\mathrm{Q}]$ works in our setting.

Proposition 4.2. One has $\mathbf{x} \in P\left(\ddot{X}_{v}^{w}\right)$ if and only if $\ddot{d}_{R}(\mathbf{x})$ and $\ddot{d} R_{\circ} \ddot{d}(\mathbf{x})$ are $\ddot{\kappa}$ invariant.

Proof. The proof of Proposition 2.2 of $[\mathrm{C}-\mathrm{Q}]$ works in our setting.

Fix $w \geq 0$ and $v \geq 2 w$. Let $\grave{X}_{v}^{w}$ and $\dot{X}_{v}^{w}$ be the image of the canonical inclusions of $X_{v}^{w}$ into $\ddot{X}_{v}^{w}$ and $\ddot{X}_{v}^{w-1}$ respectively, and let $\hat{\kappa}: \dot{X}_{v}^{w} \rightarrow \dot{X}_{v}^{w}$ be the map induced by $\ddot{\kappa}$. For $\mathbf{x}=\left(\mathbf{x}_{0}, 0\right) \in \grave{X}_{v}^{w}$ we write

$$
\grave{d}(\mathbf{x})=\left(d\left(\mathbf{x}_{0}\right), 0\right),
$$

and, for $\mathbf{y}=\left(0, \mathbf{y}_{0}\right) \in \dot{X}_{v}^{w}$, we write

$$
\dot{d}(\mathbf{y})=\left(0, d\left(\mathbf{y}_{0}\right)\right), \quad d^{\prime}(\mathbf{y})=\left(0, d^{\prime}\left(\mathbf{y}_{0}\right)\right) \quad \text { and } \quad \dot{t}(\mathbf{y})=\left(0, t\left(\mathbf{y}_{0}\right)\right) .
$$

It is immediate that $\hat{\kappa}$ coincides with $\dot{t}$. Note that $\ddot{\kappa}$ has finite order on $\ddot{d}_{R}(\ddot{X})=\dot{X}$ in each degree. In fact $\ddot{\kappa}^{w+1}=\mathrm{id}$ on $\dot{X}_{v}^{w}$. By the discussion in the page 86 of [C-Q],

$$
\begin{aligned}
& \dot{X}_{v}^{w}=\operatorname{ker}(\mathrm{id}-\hat{\kappa}) \oplus \operatorname{Im}(\mathrm{id}-\hat{\kappa}), \\
& P\left(\ddot{d}_{R}\left(\grave{X}_{v}^{w}\right)\right)=P\left(\dot{X}_{v}^{w}\right)=\operatorname{ker}(\mathrm{id}-\hat{\kappa}), \\
& P^{\perp}\left(\ddot{d}_{R}\left(\grave{X}_{v}^{w}\right)\right)=P^{\perp}\left(\dot{X}_{v}^{w}\right)=\operatorname{Im}(\mathrm{id}-\hat{\kappa}),
\end{aligned}
$$

and the maps $P_{\dot{X}_{v}^{w}}$ and $G_{\hat{X}_{v}^{w}}$, defined as the projection onto $\operatorname{ker}(\mathrm{id}-\hat{\kappa})$ associated with (7) and the Green operator for id $-\hat{\kappa}: \dot{X}_{v}^{w} \rightarrow \dot{X}_{v}^{w}$, respectively, satisfy:

$$
P_{\dot{X}_{v}^{w}}=\frac{1}{w+1} \sum_{i=0}^{w} \hat{\kappa}^{i} \quad \text { and } \quad G_{\dot{X}_{v}^{w}}=\frac{1}{w+1} \sum_{i=0}^{w}\left(\frac{w}{2}-i\right) \hat{\kappa}^{i} .
$$

Consequently, for all $\mathbf{x} \in \ddot{X}_{v}^{w}$,

$$
\begin{aligned}
& P \circ \ddot{d}_{R}(\mathbf{x})=\frac{1}{w+1} \sum_{i=0}^{w} \ddot{\kappa}^{i} \circ \ddot{d}_{R}(\mathbf{x})=\frac{1}{w+1} \ddot{B}(\mathbf{x}), \\
& G \circ \ddot{d}_{R}(\mathbf{x})=\frac{1}{w+1} \sum_{i=0}^{w}\left(\frac{w}{2}-i\right) \ddot{\kappa}^{i} \circ \ddot{d}_{R}(\mathbf{x}) .
\end{aligned}
$$


The formula (11) has the following consequences: it implies that

$$
\ddot{B}\left(P^{\perp}(\ddot{X})\right)=0 .
$$

Using this, we obtain that $\ddot{B}(\mathbf{x})=(w+1) \ddot{d}_{R}(P(\mathbf{x}))$ for all $\mathbf{x} \in \ddot{X}_{v}^{w}$. So, since $\left(P^{\perp}(\ddot{X}), \ddot{d}_{R}\right)$ is acyclic,

$$
H_{*}(P(\ddot{X}), \ddot{B})=H_{*}\left(P(\ddot{X}), \ddot{d}_{R}\right)=H_{*}\left(\ddot{X}, \ddot{d}_{R}\right)=0 .
$$

In the terminology of $\mathrm{C}$-Q this says that $(P(\ddot{X}), \ddot{d}, \ddot{b}, \ddot{B})$ is $\ddot{B}$-acyclic. Lastly, (10) combined with (12) and the second formula of (6), allows us to obtain an explicit formula for $P$. In fact, for $\mathbf{x} \in \dot{X}_{v}^{w}$, this is given by (10). Then, assume that $\mathbf{x} \in \grave{X}_{v}^{w}$. Since by (12), $G_{\circ} \ddot{d}_{R}(\mathbf{x}) \in \dot{X}_{v}^{w}$, we have:

$$
G \circ \ddot{d}_{\circ} \ddot{d}_{R}(\mathbf{x})=\ddot{d} \circ G \circ \ddot{d}_{R}(\mathbf{x})=\dot{d}^{\prime} \circ G \circ \ddot{d}_{R}(\mathbf{x})+s w \circ(\mathrm{id}-\dot{t}) \circ G \circ \ddot{d}_{R}(\mathbf{x}),
$$

where $s w: \dot{X}_{v}^{w} \rightarrow \grave{X}_{v}^{w}$ is the map defined by $s w(0, \mathbf{x})=(\mathbf{x}, 0)$. Using this, the second formula of (6), and the fact that $t_{\circ} \ddot{\kappa}^{i} \circ \ddot{d}_{R}(\mathbf{x})=\ddot{\kappa}^{i+1} \circ \ddot{d}_{R}(\mathbf{x})$, we obtain:

$$
\begin{aligned}
P(\mathbf{x}) & =\mathbf{x}-G \circ \ddot{d}_{R} \circ \grave{d}_{0}(\mathbf{x})-G \circ \ddot{d}_{\circ} \ddot{d}_{R}(\mathbf{x}) \\
& =\mathbf{x}-\frac{1}{w+2} \sum_{i=0}^{w+1}\left(\frac{w+1}{2}-i\right) \ddot{\kappa}^{i} \circ \ddot{d}_{R} \circ \grave{d}(\mathbf{x})+\frac{1}{w+1} \sum_{i=0}^{w}\left(\frac{w}{2}-i\right) \dot{d}^{\prime} \circ \ddot{\kappa}^{i} \circ \ddot{d}_{R}(\mathbf{x}) \\
& -\frac{1}{w+1} \sum_{i=0}^{w}\left(\frac{w}{2}-i\right) s w \circ\left(\mathrm{id}-\grave{t}^{\circ} \circ \ddot{\kappa}^{\circ} \circ \ddot{d}_{R}(\mathbf{x})\right. \\
& =\frac{1}{w+1} s w \circ \ddot{B}_{n}(\mathbf{x})-\frac{1}{w+2} \sum_{i=0}^{w+1}\left(\frac{w+1}{2}-i\right) \ddot{\kappa}^{i} \circ \ddot{d}_{R} \circ \grave{d}(\mathbf{x}) \\
& +\frac{1}{w+1} \sum_{i=0}^{w}\left(\frac{w}{2}-i\right) \dot{d}^{\prime} \circ \ddot{\kappa}^{i} \circ \ddot{d}_{R}(\mathbf{x}) .
\end{aligned}
$$

We now consider the chain complex $(\ddot{X}, \ddot{b}, \ddot{d})$ and denote by $\operatorname{ker}(\ddot{B}), \operatorname{Im}(\ddot{B})$ the kernel and image of $\ddot{B}$ on $\ddot{X}$. These are subcomplexes of $(\ddot{X}, \ddot{b}, \ddot{d})$. By (13) and (14), we have $\operatorname{ker}(\ddot{B}) / \operatorname{Im}(\ddot{B})=P^{\perp}(\ddot{X})$. Consequently,

$$
H_{*}(\operatorname{ker}(\ddot{B}) / \operatorname{Im}(\ddot{B}), \ddot{b}, \ddot{d})=0 .
$$

That is, the double mixed complex $(\ddot{X}, \ddot{d}, \ddot{b}, \ddot{B})$ has the Connes property $([\mathrm{C}-\mathrm{Q}])$.

Let us define the reduced cyclic complex $\bar{C}_{X}^{\lambda}$ to be the quotient double complex $\bar{C}_{X}^{\lambda}=\ddot{X} / \operatorname{ker}(\ddot{B})$. It is easy to check that $\bar{C}_{X}^{\lambda}=\frac{P(\ddot{X}) \oplus P^{\perp}(\ddot{X})}{\operatorname{lm}(\ddot{B}) \oplus P^{\perp}(\ddot{X})}=\frac{P(\ddot{X})}{\operatorname{lm}(\ddot{B})}$ and that $\breve{B}$ induces the isomorphism of complexes $\operatorname{Tot}\left(\bar{C}_{X}^{\lambda}\right)[1] \simeq \operatorname{Im}(\breve{B})$. So, we have a short exact sequence of double complexes

$$
0 \longrightarrow \operatorname{Tot}\left(\bar{C}_{X}^{\lambda}\right)[1] \stackrel{i}{\longrightarrow} \operatorname{Tot}(P)(\breve{X}) \stackrel{j}{\longrightarrow} \operatorname{Tot}\left(\bar{C}_{X}^{\lambda}\right) \longrightarrow 0,
$$

where $j$ is the canonical surjection and $i$ is induced by $\ddot{B}$.

4.4. A description of $P(\ddot{X})$. The aim of this subsection is to obtain a precise description of the double mixed complex $(P(\ddot{X}), \ddot{d}, \ddot{b}, \ddot{B})$. The main result are Theorem 4.3 and Proposition 4.4 We relegate their proofs to Appendix B.

Take $\mathbf{x}=\left(\mathbf{x}_{0}, \mathbf{x}_{1}\right) \in \ddot{X}_{v}^{w}$, with $\mathbf{x}_{0} \in X_{v}^{w}$ and $\mathbf{x}_{1} \in X_{v}^{w+1}$. By Proposition 4.2 we know that $\mathbf{x} \in P(\ddot{X})$ if and only if $\mathbf{x}_{0}$ and $d\left(\mathbf{x}_{0}\right)+($ id $-t)\left(\mathbf{x}_{1}\right)$ are $t$-invariant. From this it follows immediately that if $\mathbf{x} \in P(\ddot{X})$, then $\left(\mathbf{x}_{0}, \mathbf{x}_{1}^{\prime}\right) \in P(\ddot{X})$ for all $\mathbf{x}_{1}^{\prime} \in X_{v}^{w+1}$ such that $\mathbf{x}_{1}^{\prime}-\mathbf{x}_{1}$ is $t$-invariant. Conversely, if $\mathbf{x}$ and $\left(\mathbf{x}_{0}, \mathbf{x}_{1}^{\prime}\right)$ belong to $P(\ddot{X})$, then (id $-t)\left(\mathbf{x}_{1}^{\prime}-\mathbf{x}_{1}\right)$ is $t$-invariant, but this implies that $(\mathrm{id}-t)\left(\mathbf{x}_{1}^{\prime}-\mathbf{x}_{1}\right)=0$. 
In other words, that $\mathbf{x}_{1}^{\prime}-\mathbf{x}_{1}$ is $t$-invariant. In particular $\left(0, \mathbf{x}_{1}\right) \in P(\ddot{X})$ if and only if $\mathbf{x}_{1}$ is $t$-invariant. We let ${ }^{t} P\left(\ddot{X}_{v}^{w}\right)$ denote the set of all elements of the shape $\left(0, \mathbf{x}_{1}\right) \in \ddot{X}_{v}^{w}$ with $\mathbf{x}_{1}$ a $t$-invariant element. It is immediate that $\left({ }^{t} P(\ddot{X}),-b, d^{\prime}\right)$ is a subcomplex of $(P(\ddot{X}), \ddot{b}, \ddot{d})$.

We assert that if $\mathbf{x} \in X_{v}^{w}$ is $t$-invariant, then

$$
\left(\mathbf{x},-\sigma^{\prime} \circ d(\mathbf{x})\right) \in P(\ddot{X}) .
$$

This follows immediately from the equality $($ id $-t) \circ \sigma^{\prime}(\mathbf{y})=\mathbf{y}-\frac{1}{w+2} N(\mathbf{y})$, which was obtained in the proof of Lemma 3.3

Recall from Theorem 3.4 that $\bar{X}_{v}^{w}$ is the cokernel of id $-t: X_{v}^{w} \rightarrow X_{v}^{w}$. Let $\widetilde{X}_{v}^{w}=\bar{X}_{v}^{w} \oplus \bar{X}_{v}^{w+1}$. Consider the diagram

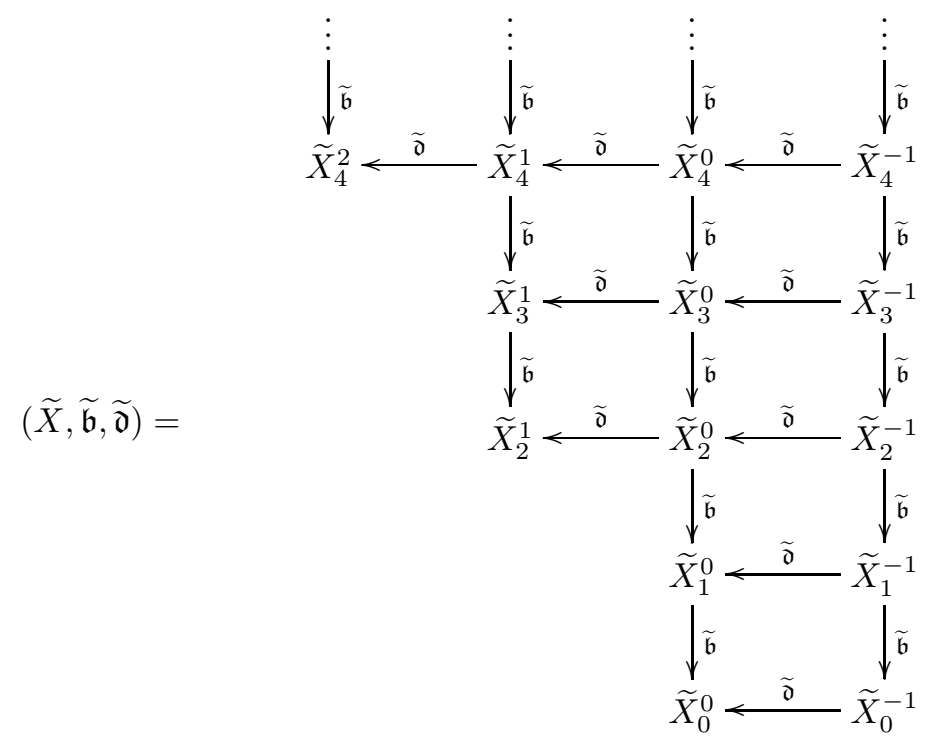

where the maps $\widetilde{\mathfrak{b}}: \widetilde{X}_{v}^{w} \rightarrow \widetilde{X}_{v-1}^{w}$ and $\widetilde{\mathfrak{d}}: \widetilde{X}_{v}^{w} \rightarrow \widetilde{X}_{v}^{w+1}$ are defined by

$$
\widetilde{\mathfrak{b}}(\mathbf{x}, \mathbf{y})=(\bar{b}(\mathbf{x}),-\bar{b}(\mathbf{y})) \text { and } \widetilde{\mathfrak{d}}(\mathbf{x}, \mathbf{y})=(\bar{d}(\mathbf{x}),-\bar{d}(\mathbf{y})) \text {, }
$$

respectively. Let $\mathfrak{p}: X_{v}^{w} \rightarrow \bar{X}_{v}^{w}$ be the map defined by

$$
\mathfrak{p}(\mathbf{x})=\frac{n-i_{w}+2}{v+2}[\mathbf{x}] \quad \text { for each elementary tensor } \mathbf{x} \in X_{v}^{w},
$$

where $i_{w}$ is the last index such that $x_{i_{w}} \in M$ and $[\mathbf{x}]$ denotes the class of $\mathbf{x}$ in $\bar{X}_{v}^{w}$. Let $\bar{N}: \bar{X}_{v}^{w} \rightarrow X_{v}^{w}$ be the map induced by $N$. It is immediate that $\mathfrak{p}$ is a retraction of $\bar{N}$. Given a $t$-invariant element $\mathbf{x} \in X_{v}^{w}$, let

$$
\Upsilon(\mathbf{x})=(-\mathrm{id}+\bar{N} \circ \mathfrak{p}) \circ \sigma^{\prime} \circ d(\mathbf{x}) \in X_{v}^{w+1} .
$$

Let ${ }^{e} \widetilde{\xi}: \widetilde{X}_{v}^{w} \rightarrow \widetilde{X}_{v-1}^{w}$ and ${ }^{e} \widetilde{\varsigma}: \widetilde{X}_{v}^{w} \rightarrow \widetilde{X}_{v}^{w+1}$ be the maps defined by

$$
e^{e} \widetilde{\xi}(\mathbf{x}, \mathbf{y})=\left(0,{ }^{e} \bar{\xi}(\mathbf{x})\right) \quad \text { and } \quad{ }^{e} \widetilde{\varsigma}(\mathbf{x}, \mathbf{y})=\left(0,{ }^{e} \bar{\zeta}(\mathbf{x})\right),
$$

where ${ }^{e} \bar{\xi}: \bar{X}_{v}^{w} \rightarrow \bar{X}_{v-1}^{w+1}$ and ${ }^{e} \bar{\varsigma}: \bar{X}_{v}^{w} \rightarrow \bar{X}_{v}^{w+2}$ are the maps given by

$$
{ }^{e} \bar{\xi}(\mathbf{x})=-\frac{1}{w+1} \mathfrak{p} \circ \sigma^{\prime} \circ d \circ \bar{N} \circ \bar{b}(\mathbf{x})-\frac{1}{w+1} \bar{b} \circ \mathfrak{p} \circ \sigma^{\prime} \circ d \circ \bar{N}(\mathbf{x})
$$

and

$$
{ }^{e} \bar{\varsigma}(\mathbf{x})=\frac{1}{w+1} \bar{d} \circ \mathfrak{p} \circ \sigma^{\prime} \circ d \circ \bar{N}(\mathbf{x})+\frac{1}{w+2} \mathfrak{p} \circ \sigma^{\prime} \circ d \circ \bar{N} \circ \bar{d}(\mathbf{x}),
$$


respectively.

Theorem 4.3. Let $\widetilde{\mathfrak{B}}: \widetilde{X}_{v}^{w} \rightarrow \widetilde{X}_{v+1}^{w}$ be the map defined by $\widetilde{\mathfrak{B}}(\mathbf{x}, \mathbf{y})=(0, \mathbf{x})$. The diagrams $\left(\widetilde{X}, \widetilde{\mathfrak{d}}, \widetilde{\mathfrak{b}}+{ }^{e} \widetilde{\xi}, \widetilde{\mathfrak{B}}\right)$ and $\left(\widetilde{X}, \widetilde{\mathfrak{d}}+{ }^{e} \widetilde{\varsigma}, \widetilde{\mathfrak{b}}, \widetilde{\mathfrak{B}}\right)$ are mixed double complexes and the maps

$\Psi:\left(\widetilde{X}, \widetilde{\mathfrak{d}}, \widetilde{\mathfrak{b}}+{ }^{e} \widetilde{\xi}, \widetilde{\mathfrak{B}}\right) \rightarrow(P(\ddot{X}), \ddot{d}, \ddot{b}, \ddot{B}) \quad$ and $\quad \Lambda:\left(\widetilde{X}, \widetilde{\mathfrak{d}}, \widetilde{\mathfrak{b}}+{ }^{e} \widetilde{\xi}, \widetilde{\mathfrak{B}}\right) \rightarrow\left(\widetilde{X}, \widetilde{\mathfrak{d}}+{ }^{e} \widetilde{\varsigma}, \widetilde{\mathfrak{b}}, \widetilde{\mathfrak{B}}\right)$, defined by $\Psi_{v}^{-1}(0, \mathbf{y})=\Lambda_{v}^{-1}(0, \mathbf{y})=(0, \mathbf{y})$, and

$$
\begin{aligned}
& \Psi_{v}^{w}(\mathbf{x}, \mathbf{y})=\frac{1}{w+1}(\bar{N}(\mathbf{x}), \Upsilon \circ \bar{N}(\mathbf{x}))+(0, \bar{N}(\mathbf{y})), \\
& \Lambda_{v}^{w}(\mathbf{x}, \mathbf{y})=(\mathbf{x}, \mathbf{y})+\frac{1}{w+1}\left(0, \mathfrak{p} \circ \sigma^{\prime} \circ d \circ \bar{N}(\mathbf{x})\right),
\end{aligned}
$$

for $w \geq 0$, are isomorphisms of double mixed complexes.

Proof. See Appendix B.

We now give a formula for ${ }^{e} \bar{\zeta}$.

Proposition 4.4. Let $\mathbf{x}_{0}^{n} \in X_{v}^{w}$ be an elementary tensor, let $0=i_{0}<\cdots<i_{w} \leq n$ be the indices such that $x_{i_{j}} \in M$ and let $i_{w+1}=n+1$. Given $0 \leq \alpha \leq n$ we let $j(\alpha)$ denote the number defined by $i_{j(\alpha)} \leq \alpha<i_{j(\alpha)+1}$. We have:

$$
e_{\bar{\zeta}}\left(\left[\mathbf{x}_{0}^{n}\right]\right)=\sum_{\alpha<\beta} \lambda_{\alpha \beta}^{(w)}\left[F_{\alpha} \circ F_{\beta}\left(\mathbf{x}_{0}^{n}\right)\right]
$$

where $\left[F_{\alpha}{ }^{\circ} F_{\beta}\left(\mathbf{x}_{0}^{n}\right)\right]$ denotes the class of $F_{\alpha}{ }^{\circ} F_{\beta}\left(\mathbf{x}_{0}^{n}\right)$ in $X_{v}^{w+2}$ and

$$
\lambda_{\alpha \beta}^{(w)}=\frac{2(j(\beta)-j(\alpha))}{(w+1)(w+2)(w+3)}-\frac{1}{(w+2)(w+3)} .
$$

Proof. See Appendix B.

Let $i: \bar{X}_{v}^{w+1} \rightarrow \widetilde{X}_{v}^{w}$ and $\pi: \widetilde{X}_{v}^{w} \rightarrow \bar{X}_{v}^{w}$ be the canonical maps. The short exact sequence of double complexes

$$
0 \longrightarrow\left(\bar{X}_{*}^{*+1}, \bar{b}, \bar{d}\right) \stackrel{i}{\longrightarrow}\left(\widetilde{X}_{*}^{*}, \widetilde{\mathfrak{b}}, \widetilde{\mathfrak{d}}+{ }^{e} \widetilde{\varsigma}\right) \stackrel{\pi}{\longrightarrow}\left(\bar{X}_{*}^{*}, \bar{b}, \bar{d}\right) \longrightarrow 0 .
$$

splits in each level via the maps $s: \bar{X}_{v}^{w} \rightarrow \widetilde{X}_{v}^{w}$ and $r: \widetilde{X}_{v}^{w} \rightarrow \bar{X}_{v}^{w+1}$, given by $s(\mathbf{x})=(\mathbf{x}, 0)$ and $r(\mathbf{x}, \mathbf{y})=\mathbf{y}$. From this it follows immediately that the connection map of the homology long exact sequence associated with (16) is induced by the morphism of double complexes ${ }^{e} \bar{\zeta}:\left(\bar{X}_{*}^{*}, \bar{b}, \bar{d}\right) \rightarrow\left(\bar{X}_{*}^{*-2}, \bar{b}, \bar{d}\right)$.

Proposition 4.5. The maps

$$
\begin{aligned}
& S_{n}: \mathrm{HC}_{n}(E, M) \rightarrow \mathrm{HC}_{n-2}(E, M), \\
& B_{n}: \mathrm{HC}_{n}(E, M) \rightarrow \mathrm{HH}_{n+1}(E, M), \\
& i_{n}: \mathrm{HH}_{n}(E, M) \rightarrow \mathrm{HC}_{n}(E, M),
\end{aligned}
$$

are induced by $-{ }^{e} \bar{\varsigma}, i$ and $\pi$, respectively.

Proof. Left to the reader.

Let $C$ be a $k$-algebra and let $M$ be a two-sided ideal of $C$. In G2 was proved that if $M^{m+1}=0$, then

$$
S^{m(n+1)}: \mathrm{HC}_{n+2 m(n+1)}(C, M) \rightarrow \mathrm{HC}_{n}(C, M)
$$

is the zero map. Actually, arguing as in the proof of Theorem 4.6 it is easy to see that if the previous formula holds when $m=1$, then it is valid for all $m$ whenever $M^{2^{m}}=0$. Next, we give a theorem that improves this result. 
Theorem 4.6. If $M^{2^{m}}=0$, then

$$
S^{m([n / 2]+1)}: \mathrm{HC}_{n+2 m([n / 2]+1)}(C, M) \rightarrow \mathrm{HC}_{n}(C, M) \quad(n \geq 0)
$$

is the zero map, where $[n / 2]$ denotes the integer part of $n / 2$.

Proof. We make the proof by induction on $m$. For $m=1$ the theorem follows from Proposition 4.5 Assume that $m>1$ and that the corollary is valid for $m-1$. Let $l=\left[\frac{n}{2}\right]+1$. Consider the commutative diagram with exact rows

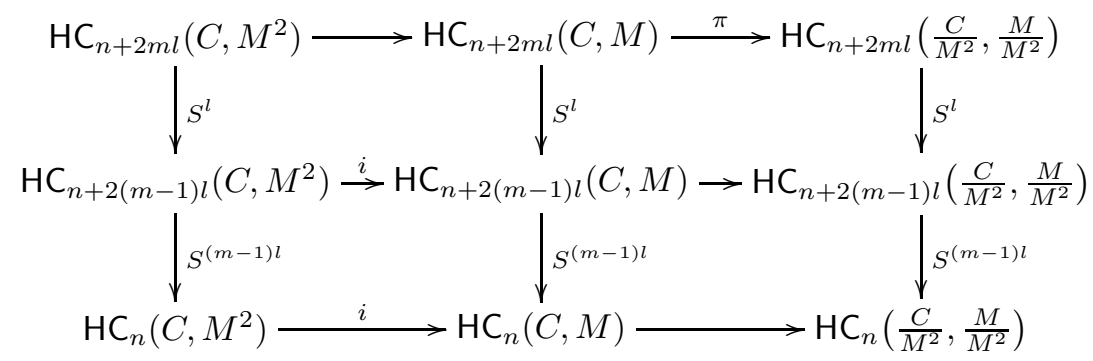

where $i$ and $\pi$ are the canonical maps. Let $\mathbf{x} \in \mathrm{HC}_{n+2 m l}(C, M)$. By the case $m=1$, we know that $S^{l}(\pi(\mathbf{x}))=0$. So, there exists $\mathbf{y} \in \mathrm{HC}_{n+2(m-1) l}\left(C, M^{2}\right)$ such that $S^{l}(\mathbf{x})=i(\mathbf{y})$. Since $\left(M^{2}\right)^{2^{m-1}}=M^{2^{m}}=0$ we can apply the inductive hypothesis to conclude that $S^{m l}(\mathbf{x})=i\left(S^{(m-1) l}\left(S^{l}(\mathbf{y})\right)\right)=0$.

Note that Theorem 4.6 implies that if $M^{2}=0$, then the maps

$$
S_{2}: \mathrm{HC}_{2}(C, M) \rightarrow \mathrm{HC}_{0}(C, M) \text { and } S_{3}: \mathrm{HC}_{3}(C, M) \rightarrow \mathrm{HC}_{1}(C, M)
$$

are zero.

\subsection{The connection map for the Hochschild homology revised. Let}

$$
\widehat{\delta}^{3}: A \otimes \bar{A}^{\otimes n} \rightarrow \bar{X}_{n-1}^{1} \quad \text { and } \quad \widetilde{\delta}^{3}: A \otimes \bar{A}^{\otimes n} \rightarrow \bar{X}_{n-1}^{1}
$$

be the maps defined by

$$
\begin{aligned}
& \widehat{\delta}^{3}(\mathbf{a})=\sum_{j=2}^{n-1} \frac{n+1-2 j}{2(n+1)} F_{0 j}(\mathbf{a})+\sum_{i=0}^{n-1} \frac{i+1-n}{n+1} F_{i n}(\mathbf{a})+\sum_{0<i<j<n} \frac{i-j}{n+1} F_{i j}(\mathbf{a}), \\
& \widetilde{\delta}^{3}(\mathbf{a})=-\frac{1}{2} \sum_{0<i<j \leq n} F_{i j}(\mathbf{a}),
\end{aligned}
$$

where $F_{i j}(\mathbf{a})$ is the class of $t_{\circ} F_{i} \circ F_{j}(\mathbf{a})$ in $\bar{X}_{n-1}^{1}$.

Proposition 4.7. The connection map $\delta_{n}: \mathrm{HH}_{n}(A) \rightarrow \mathrm{HH}_{n-1}(E, M)$ associated with the short exact sequence (3) is induced by the morphisms of complexes

$$
\widehat{\delta}:\left(A \otimes \bar{A}^{\otimes *}, b\right) \rightarrow \operatorname{Tot}\left(\widetilde{X}, \widetilde{\mathfrak{d}}, \widetilde{\mathfrak{b}}+{ }^{e} \widetilde{\xi}\right) \quad \text { and } \quad \widetilde{\delta}:\left(A \otimes \bar{A}^{\otimes *}, b\right) \rightarrow \operatorname{Tot}\left(\widetilde{X}, \widetilde{\mathfrak{d}}+{ }^{e} \widetilde{\varsigma}, \widetilde{\mathfrak{b}}\right) \text {, }
$$

given by

$$
\widehat{\delta}(\mathbf{a})=\left(\delta^{2}(\mathbf{a}), \delta^{1}(\mathbf{a}), \widehat{\delta}^{3}(\mathbf{a}), 0,0, \ldots\right) \quad \text { and } \quad \widetilde{\delta}(\mathbf{a})=\left(\delta^{2}(\mathbf{a}), \delta^{1}(\mathbf{a}), \widetilde{\delta}^{3}(\mathbf{a}), 0,0, \ldots\right),
$$

where we are writing

$$
\begin{aligned}
\operatorname{Tot}\left(\left(\widetilde{X}, \widetilde{\mathfrak{d}}, \widetilde{\mathfrak{b}}+{ }^{e} \widetilde{\xi}\right)\right)_{n-1} & =\operatorname{Tot}\left(\left(\widetilde{X}, \widetilde{\mathfrak{d}}+{ }^{e} \widetilde{\varsigma}, \widetilde{\mathfrak{b}}\right)\right)_{n-1} \\
& =\widetilde{X}_{n-2}^{-1} \oplus \widetilde{X}_{n-1}^{0} \oplus \widetilde{X}_{n}^{1} \oplus \widetilde{X}_{n+1}^{2} \oplus \widetilde{X}_{n+2}^{3} \oplus \cdots \\
& \simeq \bar{X}_{n-2}^{0} \oplus \bar{X}_{n-1}^{0} \oplus \bar{X}_{n-1}^{1} \oplus \bar{X}_{n}^{1} \oplus \bar{X}_{n}^{2} \oplus \cdots
\end{aligned}
$$

(here we are identifying $\widetilde{X}_{n-2}^{-1}=\bar{X}_{n-2}^{-1} \oplus \bar{X}_{n-2}^{0}$ with $\bar{X}_{n-2}^{0}$ ). 
Proof. Let $\breve{\delta}$ be the morphism introduced in Proposition 3.7 By Theorem 4.3 to check the assertions it suffices to show that $\operatorname{Tot}(\Psi)_{n-1} \circ \widehat{\delta}_{n}=\operatorname{Tot}(P)_{n} \circ \breve{\delta}_{n}$ and $\operatorname{Tot}(\Lambda)_{n-1}{ }^{\circ} \widehat{\delta}_{n}=\widetilde{\delta}_{n}$. In other words we must prove that

$$
\begin{aligned}
& \Psi^{-1}\left(0, \delta^{2}(\mathbf{a})\right)=\left(0, \delta^{2}(\mathbf{a})\right), \\
& \Psi^{0}\left(\delta^{1}(\mathbf{a}), \widehat{\delta}_{n}^{3}(\mathbf{a})\right)=P\left(\delta^{1}(\mathbf{a}), \delta^{3}(\mathbf{a})\right), \\
& \Lambda^{-1}\left(0, \delta^{2}(\mathbf{a})\right)=\left(0, \delta^{2}(\mathbf{a})\right), \\
& \Lambda^{0}\left(\delta^{1}(\mathbf{a}), \widehat{\delta}^{3}(\mathbf{a})\right)=\left(\delta^{1}(\mathbf{a}), \widetilde{\delta}^{3}(\mathbf{a})\right),
\end{aligned}
$$

where $\mathbf{a} \in A \otimes \bar{A}^{\otimes n}$ and $\delta^{1}(\mathbf{a}), \delta^{2}(\mathbf{a})$ and $\delta^{3}(\mathbf{a})$ are as in Subsection 3.2 Equalities (17) and (19) are immediate. To check the equality (18) we first compute $\breve{P}\left(\delta^{1}(\mathbf{a}), \delta^{3}(\mathbf{a})\right)$. Since, by (15) we know that $\left(\delta^{1}(\mathbf{a}),-\sigma^{\prime} \circ d^{\circ} \delta^{1}(\mathbf{a})\right) \in P(\ddot{X})$ and $P$ is a projection, we have:

$$
\begin{aligned}
P\left(\delta^{1}(\mathbf{a}), \delta^{3}(\mathbf{a})\right) & =P\left(\delta^{1}(\mathbf{a}),-\sigma^{\prime} \circ d^{1} \delta^{1}(\mathbf{a})\right)+P\left(0,\left(\delta^{3}+\sigma^{\prime} \circ d \circ \delta^{1}\right)(\mathbf{a})\right) \\
& =\left(\delta^{1}(\mathbf{a}),-\sigma^{\circ} \circ d^{1} \delta^{1}(\mathbf{a})\right)+P \circ \ddot{d}_{R} \circ\left(\left(\delta^{3}+\sigma^{\circ} \circ d^{\circ} \delta^{1}\right)(\mathbf{a}), 0\right) \\
& =\left(\delta^{1}(\mathbf{a}), \frac{1}{2} \delta^{3}(\mathbf{a})+\frac{1}{2} t \circ \delta^{3}(\mathbf{a})-\frac{1}{2} \sigma^{\prime} \circ \circ \circ \delta^{1}(\mathbf{a})+\frac{1}{2} t \circ \sigma^{\prime} \circ d \circ \delta^{1}(\mathbf{a})\right) .
\end{aligned}
$$

where the third equality follows from (11). Let

$$
L(\mathbf{a}):=\frac{1}{2} \delta^{3}(\mathbf{a})+\frac{1}{2} t \circ \delta^{3}(\mathbf{a})-\frac{1}{2} \sigma^{\prime} \circ d \circ \delta^{1}(\mathbf{a})+\frac{1}{2} t \circ \sigma^{\prime} \circ d \circ \delta^{1}(\mathbf{a}) .
$$

By the definition of $\Psi^{0}$, to prove equality (18), we must show that

$$
(-\mathrm{id}+\bar{N} \circ \mathfrak{p}) \circ \sigma^{\prime} \circ d \circ \delta^{1}(\mathbf{a})+\bar{N}\left(\widehat{\delta}^{3}(\mathbf{a})\right)=L(\mathbf{a}) .
$$

A direct computation shows that

$$
t \circ F_{i}^{\circ} F_{j}(\mathbf{a})= \begin{cases}F_{i+n-j} \circ{ }^{\circ} \circ F_{j}(\mathbf{a}) & \text { if } 0 \leq i<j-1<n-1, \\ F_{i+1} \circ{ }^{\circ} F_{n}(\mathbf{a}) & \text { if } 0 \leq i<n-2 \text { and } j=n,\end{cases}
$$

and

$$
t^{2} \circ F_{i} \circ F_{j}(\mathbf{a})= \begin{cases}-F_{j-i-1} \circ{ }^{\circ} F_{i}(\mathbf{a}) & \text { if } 0 \leq i<j-1<n-1, \\ -F_{n-i-2} \circ{ }^{\circ} F_{i+1}(\mathbf{a}) & \text { if } 0 \leq i<n-2 \text { and } j=n .\end{cases}
$$

Hence,

$$
\sigma^{\prime} \circ d^{\circ} \delta^{1}(\mathbf{a})=\frac{1}{2} \sum_{i=1}^{n-2} \sum_{j=0}^{n} F_{i} \circ{ }^{\circ} F_{j}(\mathbf{a})=\frac{1}{2} \sum_{0 \leq i<j \leq n} t \circ F_{i} \circ F_{j}(\mathbf{a})-\frac{1}{2} \sum_{0 \leq i<j \leq n} t^{2} \circ F_{i} \circ F_{j}(\mathbf{a}) .
$$

Thus, by the definition of $\delta^{3}$,

$$
L(\mathbf{a})=\frac{1}{2} \sum_{j=2}^{n-1} F_{0} \circ F_{j}(\mathbf{a})+\frac{1}{2} \sum_{j=2}^{n-1} t_{\circ} F_{0} \circ F_{j}(\mathbf{a})-\sum_{0 \leq i<j \leq n} t \circ F_{i} \circ F_{j}(\mathbf{a}) .
$$

and, by the definition of $\mathfrak{p}$,

$$
\begin{aligned}
\mathfrak{p} \circ \sigma^{\prime} \circ d \circ \delta^{1}(\mathbf{a}) & =\sum_{0 \leq i<j<n} \frac{j-i}{2(n+1)}\left[t \circ F_{i} \circ F_{j}(\mathbf{a})\right]+\sum_{i=0}^{n-1} \frac{n-i-1}{2(n+1)}\left[t \circ F_{i} \circ F_{n}(\mathbf{a})\right] \\
& -\sum_{0 \leq i<j<n} \frac{n-j+i+1}{2(n+1)}\left[t^{2} \circ F_{i} \circ F_{j}(\mathbf{a})\right]-\sum_{i=0}^{n-1} \frac{i+2}{2(n+1)}\left[t^{2} \circ F_{i} \circ F_{n}(\mathbf{a})\right] \\
& =\sum_{0 \leq i<j<n} \frac{2 j-2 i-n-1}{2(n+1)}\left[t \circ F_{i} \circ F_{j}(\mathbf{a})\right]+\sum_{i=0}^{n-1} \frac{n-2 i-3}{2(n+1)}\left[t \circ F_{i} \circ F_{n}(\mathbf{a})\right]
\end{aligned}
$$


where $[\mathbf{x}]$ denotes the class of $\mathbf{x} \in X_{n-1}^{1}$ in $\bar{X}_{n-1}^{1}$. Consequently,

$$
\begin{aligned}
& L(\mathbf{a})+(\mathrm{id}-\bar{N} \circ \mathfrak{p}) \circ \sigma^{\prime} \circ d^{\circ} \delta^{1}(\mathbf{a})=\sum_{j=2}^{n-1} \frac{n+1-2 j}{2(n+1)}\left(t+t^{2}\right) \circ F_{0} \circ F_{j}(\mathbf{a}) \\
& +\sum_{i=0}^{n-1} \frac{i+1-n}{n+1}\left(t+t^{2}\right) \circ F_{i} \circ F_{n}(\mathbf{a})+\sum_{0<i<j<n} \frac{i-j}{n+1}\left(t+t^{2}\right) \circ F_{i} \circ F_{j}(\mathbf{a}) .
\end{aligned}
$$

The equality (21) follows immediately from this fact. To prove equality (20) we must show that

$$
\widehat{\delta}_{n}^{3}(\mathbf{a})+\mathfrak{p} \circ \sigma^{\prime} \circ d \circ \delta^{1}(\mathbf{a})=-\frac{1}{2} \sum_{0<i<j \leq n}\left[t \circ F_{i} \circ F_{j}(\mathbf{a})\right]
$$

which can be checked by a direct computation.

\section{APPENDiX A.}

This appendix is devoted to prove Theorem 3.2 and Propositions 3.7 and 3.8

Let $E=A \ltimes_{f} M$ be a square zero extension. By definition, the Hochschild homology of $E$ relative to $M$ is the homology of the complex

$$
(\breve{\mathfrak{X}}, \breve{\mathfrak{b}})=\operatorname{ker}\left(\left(E \otimes \bar{E}^{\otimes *}, b\right) \stackrel{\pi}{\longrightarrow}\left(A \otimes \bar{A}^{\otimes *}, b\right)\right)
$$

where $\pi$ is the canonical projection. Let $\hat{\mathfrak{X}}_{v}^{w}=\left(A \otimes B_{w+1}^{n}\right) \oplus\left(M \otimes B_{w}^{n}\right)$. It is immediate that $(\breve{\mathfrak{X}}, \breve{\mathfrak{b}})$ is the total complex of the second quadrant double complex

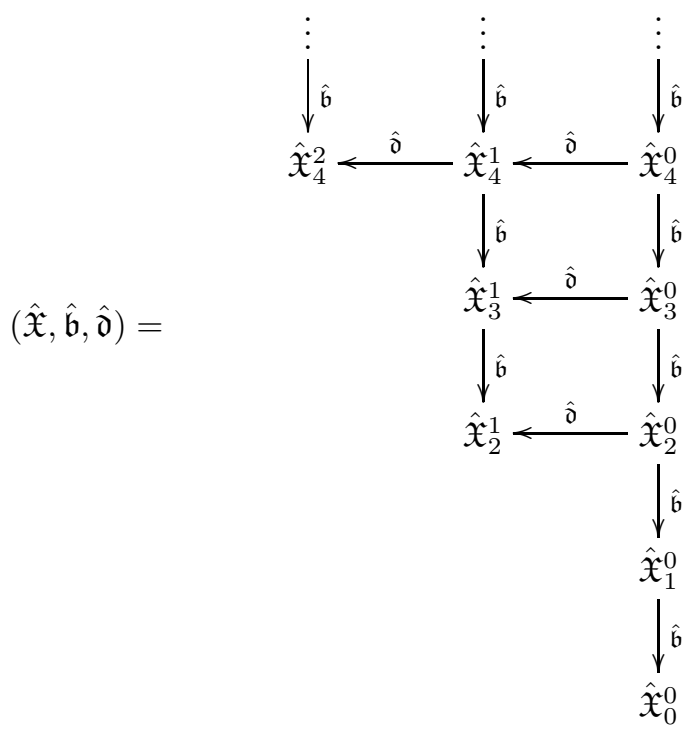

where $\hat{\mathfrak{d}}: \hat{\mathfrak{X}}_{v}^{w} \rightarrow \hat{\mathfrak{X}}_{v}^{w+1}$ is defined by $\hat{\mathfrak{d}}\left(\mathbf{x}_{0}^{n}\right)=\sum_{j=0}^{n-1} F_{j}\left(\mathbf{x}_{0}^{n}\right)+t \circ F_{n}\left(\mathbf{x}_{0}^{n}\right)$ and $\hat{\mathfrak{b}}$ is given by the same formula as the Hochschild boundary map. 
The $w$-th column $\left(\hat{\mathfrak{X}}^{w}, \hat{\mathfrak{b}}\right)$ of the above complex is the total complex of the double complex

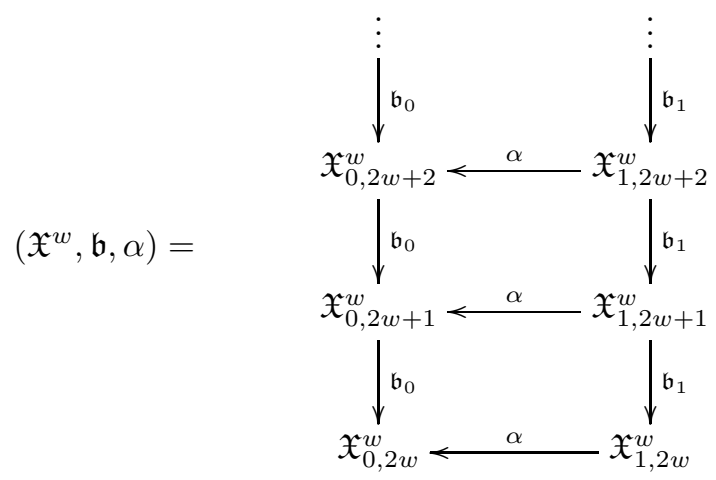

where $\mathfrak{X}_{0 v}^{w}=M \otimes B_{w}^{n}, \mathfrak{X}_{1, v-1}^{w}=A \otimes B_{w+1}^{n}, \mathfrak{b}_{0}$ is given by the same formula as the Hochschild boundary map, $\mathfrak{b}_{1}\left(\mathbf{x}_{0}^{n}\right)=\mu_{0}^{A}\left(\mathbf{x}_{0}^{n}\right)+\sum_{j=1}^{n-1} \mu_{l}\left(\mathbf{x}_{0}^{n}\right)+\mu_{n}^{A}\left(\mathbf{x}_{0}^{n}\right)$ and $\alpha\left(\mathbf{x}_{0}^{n}\right)=\mu_{0}^{M}\left(\mathbf{x}_{0}^{n}\right)+\mu_{n}^{M}\left(\mathbf{x}_{0}^{n}\right)$.

Lemma A.1. Let $\theta_{1}^{w}:\left(\mathfrak{X}_{1}^{w}, \mathfrak{b}_{1}\right) \rightarrow\left(X_{1}^{w},-b\right)$ and $\vartheta_{1}^{w}:\left(X_{1}^{w},-b\right) \rightarrow\left(\mathfrak{X}_{1}^{w}, \mathfrak{b}_{1}\right)$ be the morphisms of complexes given by

$$
\theta_{1}^{w}\left(\mathbf{x}_{0}^{n+1}\right)=\mu_{0}^{M}\left(\mathbf{x}_{0}^{n+1}\right) \quad \text { and } \quad \vartheta_{1}^{w}\left(\mathbf{x}_{0}^{n}\right)=\sum_{l=0}^{n-i\left(\mathbf{x}_{0}^{n}\right)} 1 \otimes \mathfrak{t}^{l}\left(\mathbf{x}_{0}^{n}\right),
$$

where $\mathfrak{t}\left(\mathbf{x}_{0}^{n}\right)=(-1)^{n} x_{n} \otimes \mathbf{x}_{0}^{n-1}$. Then, $\theta_{1}^{w} \circ \vartheta_{1}^{w}=\mathrm{id}$ and $\vartheta_{1}^{w} \circ \theta_{1}^{w}$ is homotopic to id. A homotopy is the family of maps $\epsilon^{w}: \mathfrak{X}_{1, v-1}^{w} \rightarrow \mathfrak{X}_{1 v}^{w}$, defined by

$$
\epsilon^{w}\left(\mathbf{x}_{0}^{n}\right)=-\sum_{l=0}^{n-i\left(\mathbf{x}_{0}^{n}\right)} 1 \otimes \mathfrak{t}^{l}\left(\mathbf{x}_{0}^{n}\right)
$$

Proof. It is immediate that $\theta_{1}^{w}$ is a morphism of chain complexes and $\theta_{1}^{w} \circ \vartheta_{1}^{w}=$ id. We claim that $\vartheta_{1}^{w}$ is also a morphism of chain complexes. Let $\mathbf{x} \in X_{1 v}^{w}$ be an elementary tensor and let $i=i(\mathbf{x})$. On one hand, a direct computation shows that

$$
\mu_{0}^{A}(1 \otimes \mathbf{x})=\mu_{n+1}^{A}\left(1 \otimes \mathfrak{t}^{n-i}(\mathbf{x})\right)=0 \quad \text { and } \quad \mu_{0}^{A}\left(1 \otimes \mathfrak{t}^{l}(\mathbf{x})\right)=-\mu_{n+1}^{A}\left(1 \otimes \mathfrak{t}^{l-1}(\mathbf{x})\right)
$$

for $1 \leq l \leq n-i$, and so

$$
\mathfrak{b}_{1}\left(\vartheta_{1}^{w}(\mathbf{x})\right)=\sum_{j=1}^{n} \sum_{l=0}^{n-i} \mu_{j}\left(1 \otimes \mathfrak{t}^{l}(\mathbf{x})\right)=-1 \otimes \sum_{j=0}^{n-1} \sum_{l=0}^{n-i} \mu_{j}\left(\mathfrak{t}^{l}(\mathbf{x})\right) .
$$

On the other hand, it is easy to see that

$$
\mathfrak{t}^{l}\left(\mu_{j}(\mathbf{x})\right)= \begin{cases}\mu_{j+l}\left(\mathfrak{t}^{l}(\mathbf{x})\right) & \text { if } 0 \leq l \leq n-i \text { and } 0 \leq j \leq n-l-1 \\ \mu_{j+l-n}\left(\mathfrak{t}^{l+1}(\mathbf{x})\right) & \text { if } 0 \leq l \leq n-i-1 \text { and } n-l \leq j \leq n\end{cases}
$$

and so

$$
\vartheta_{1}^{w}(b(\mathbf{x}))=\sum_{j=0}^{i-1} 1 \otimes \mathfrak{t}^{n-i}\left(\mu_{j}(\mathbf{x})\right)+\sum_{l=0}^{n-i-1} \sum_{j=0}^{n} 1 \otimes \mathfrak{t}^{l}\left(\mu_{j}(\mathbf{x})\right)=1 \otimes \sum_{j=0}^{n-1} \sum_{l=0}^{n-i} \mu_{j}\left(\mathfrak{t}^{l}(\mathbf{x})\right)
$$

which proves the claim. We now check that $\epsilon^{w}$ is an homotopy from $\vartheta_{1}^{w} \circ \theta_{1}^{w}$ to the identity map. Let $\mathbf{x} \in \mathfrak{X}_{1, v-1}^{w}$ be an elementary tensor and let $i=i(\mathbf{x})$. On one hand, since

$\mu_{n+1}^{A}\left(1 \otimes \mathfrak{t}^{n-i}(\mathbf{x})\right)=0 \quad$ and $\quad \mu_{0}^{A}\left(1 \otimes \mathfrak{t}^{l}(\mathbf{x})\right)=-\mu_{n+1}^{A}\left(1 \otimes \mathfrak{t}^{l-1}(\mathbf{x})\right)$ for $1 \leq l \leq n-1$, 
we have

$$
\mathfrak{b}_{1}\left(\epsilon^{w}(\mathbf{x})\right)=-\mathbf{x}-\sum_{j=1}^{n} \sum_{l=0}^{n-i} \mu_{j}\left(1 \otimes \mathfrak{t}^{l}(\mathbf{x})\right)=-\mathbf{x}+\sum_{j=0}^{n-1} \sum_{l=0}^{n-i} 1 \otimes \mu_{j}\left(\mathfrak{t}^{l}(\mathbf{x})\right) .
$$

On the other hand, using the equality

$$
\left.\left.\sum_{l=0}^{n-i-1} 1 \otimes \mathfrak{t}^{l} \mu_{n}^{A}(\mathbf{x})\right)=\sum_{l=0}^{n-i-1} 1 \otimes \mathfrak{t}^{l} \mu_{n}(\mathbf{x})\right)
$$

and the equations (22) twice, we obtain

$$
\begin{aligned}
\epsilon^{w}\left(\left(\mathfrak{b}_{1}(\mathbf{x})\right)\right. & =-\sum_{l=0}^{n-i} 1 \otimes \mathfrak{t}^{l}\left(\mu_{0}^{A}(\mathbf{x})\right)-\sum_{l=0}^{n-i-1} \sum_{j=1}^{n} 1 \otimes \mathfrak{t}^{l}\left(\mu_{j}(\mathbf{x})\right)-\sum_{j=1}^{i-1} 1 \otimes \mathfrak{t}^{n-i}\left(\mu_{j}(\mathbf{x})\right) \\
& =-\sum_{l=0}^{n-i} 1 \otimes \mathfrak{t}^{l}\left(\mu_{0}^{A}(\mathbf{x})\right)-\sum_{j=0}^{n-1} \sum_{l=0}^{n-i} 1 \otimes \mu_{j}\left(\mathfrak{t}^{l}(\mathbf{x})\right)+\sum_{l=0}^{n-i} 1 \otimes \mu_{l}\left(\mathfrak{t}^{l}(\mathbf{x})\right) .
\end{aligned}
$$

Since $\mathfrak{t}^{l}\left(\mu_{0}(\mathbf{x})\right)-\mu_{l}\left(\mathfrak{t}^{l}(\mathbf{x})\right)=\mathfrak{t}^{l}\left(\mu_{0}(\mathbf{x})\right)-\mathfrak{t}^{l}\left(\mu_{0}^{A}(\mathbf{x})\right)=\mathfrak{t}^{l}\left(\mu_{0}^{M}(\mathbf{x})\right)$, we have

$$
\mathfrak{b}_{1}\left(\epsilon^{w}(\mathbf{x})\right)+\epsilon^{w}\left(\left(\mathfrak{b}_{1}(\mathbf{x})\right)=-\mathbf{x}+\sum_{l=0}^{n-i} 1 \otimes \mathfrak{t}^{l}\left(\mu_{0}^{M}(\mathbf{x})\right)=-\mathbf{x}+\vartheta_{1}^{w}\left(\theta_{1}^{w}(\mathbf{x})\right)\right.
$$

which proves that $\epsilon^{w}$ is a homotopy from $\vartheta_{1}^{w} \circ \theta_{1}^{w}$ to id.

Lemma A.2. For $w \geq 0$, let $\tau_{0}^{1}\left(\mathcal{X}^{w}\right)$ be the double diagram with two columns $\left(X^{w}, b\right) \stackrel{\text { id }-t}{\longleftarrow}\left(X^{w},-b\right)$. The following assertions hold:

(1) $\tau_{0}^{1}\left(\mathcal{X}^{w}\right)$ is a double complex.

(2) The map $\vartheta^{w}: \tau_{0}^{1}\left(\mathcal{X}^{w}\right) \rightarrow\left(\mathfrak{X}^{w}, \mathfrak{b}, \alpha\right)$, where $\vartheta_{0}^{w}: X_{v}^{w} \rightarrow \mathfrak{X}_{v}^{w}$ is the identity map and $\vartheta_{1}^{w}: X_{v-1}^{w} \rightarrow \mathfrak{X}_{v-1}^{w}$ is as in Lemma A.1 is a morphism of double complexes.

(3) The map $\hat{\theta}^{w}:\left(\hat{\mathfrak{X}}^{w}, \hat{\mathfrak{b}}\right) \rightarrow \operatorname{Tot}\left(\tau_{0}^{1}\left(\mathcal{X}^{w}\right)\right)$, defined by

$$
\hat{\theta}^{w}(\mathbf{x}, \mathbf{y})=\left(\mathbf{x}+t(\mathbf{y}), \mu_{0}^{M}(\mathbf{y})\right),
$$

is a morphism of complexes.

(4) Let $\hat{\vartheta}^{w}: \operatorname{Tot}\left(\tau_{0}^{1}\left(\mathcal{X}^{w}\right)\right) \rightarrow\left(\hat{\mathfrak{X}}^{w}, \hat{\mathfrak{b}}\right)$ be the map induced by $\vartheta^{w}$. It is true that $\hat{\theta}^{w} \circ \hat{\vartheta}^{w}=\mathrm{id}$ and $\hat{\vartheta}^{w} \hat{\theta}^{w}$ is homotopic to the identity map. A homotopy is the family of maps

$$
\hat{\epsilon}^{w}: \mathfrak{X}_{0 v}^{w} \oplus \mathfrak{X}_{1, v-1}^{w} \rightarrow \mathfrak{X}_{0, v+1}^{w} \oplus \mathfrak{X}_{1 v}^{w},
$$

defined by $\hat{\epsilon}^{w}(\mathbf{x}, \mathbf{y})=\left(0, \epsilon^{w}(\mathbf{y})\right)$, where $\epsilon^{w}$ is the homotopy introduced in Lemma A.1

Proof. By Lemma A.1 there is a special deformation retract

$$
\left(X_{*}^{w} \oplus X_{*-1}^{w}, b \oplus-b\right) \underset{\text { id } \oplus \theta_{1}^{w}}{\text { id } \oplus \vartheta_{1}^{w}}\left(\mathfrak{X}_{0 *}^{w} \oplus \mathfrak{X}_{1, *-1}^{w}, \mathfrak{b}_{0} \oplus \mathfrak{b}_{1}\right),
$$

with homotopy $\hat{\epsilon}^{w}: \mathfrak{X}_{0 *}^{w} \oplus \mathfrak{X}_{1, *-1}^{w} \rightarrow \mathfrak{X}_{0, *+1}^{w} \oplus \mathfrak{X}_{1 *}^{w}$, given by $\hat{\epsilon}^{w}(\mathbf{x}, \mathbf{y})=\left(0, \epsilon^{w}(\mathbf{y})\right)$. Applying the perturbation lemma to this endowed with the perturbation $\alpha$, we obtain a special deformation retract

$$
\widehat{\operatorname{Tot}}\left(\tau_{0}^{1}\left(\mathcal{X}^{w}\right)\right) \underset{\widetilde{\vartheta}^{w}}{\stackrel{\tilde{\theta}^{w}}{\rightleftarrows}}\left(\hat{\mathfrak{X}}^{w}, \hat{\mathfrak{b}}\right),
$$


with homotopy $\widetilde{\epsilon}^{w}: \mathfrak{X}_{0 *}^{w} \oplus \mathfrak{X}_{1, *-1}^{w} \rightarrow \mathfrak{X}_{0, *+1}^{w} \oplus \mathfrak{X}_{1 *}^{w}$. To finish the proof it remains to check that

$$
\widehat{\operatorname{Tot}}\left(\tau_{0}^{1}\left(\mathcal{X}^{w}\right)\right)=\operatorname{Tot}\left(\tau_{0}^{1}\left(\mathcal{X}^{w}\right)\right), \quad \widetilde{\vartheta}^{w}=\hat{\vartheta}^{w}, \quad \widetilde{\theta}^{w}=\hat{\theta}^{w} \quad \text { and } \quad \widetilde{\epsilon}^{w}=\hat{\epsilon}^{w},
$$

which follow easily from the fact that id $-t=\alpha \circ \vartheta_{1}^{w}$ and $\alpha \circ \epsilon^{w}=t$.

The first item of the following lemma is part of item (1) of Theorem 3.2

Lemma A.3. The following assertions hold:

(1) The diagram $(\hat{X}, \hat{b}, \hat{d})$, introduced above Theorem 3.2 is a double complex.

(2) The map $\hat{\vartheta}:(\hat{X}, \hat{b}, \hat{d}) \rightarrow(\hat{\mathfrak{X}}, \hat{\mathfrak{b}}, \hat{\mathfrak{d}})$, obtained by gluing the maps $\hat{\vartheta}^{w}$ introduced in Lemma A.2 is a morphism of double complexes.

(3) Recall from the proof of Lemma 3.1] that $(\breve{X}, \breve{b})=\operatorname{Tot}(\hat{X}, \hat{b}, \hat{d})$ and from the beginning of this section that $(\breve{\mathfrak{X}}, \breve{\mathfrak{b}})=\operatorname{Tot}(\hat{\mathfrak{X}}, \hat{\mathfrak{b}}, \hat{\mathfrak{d}})$. For $w \geq 0$ and $v \geq 2 w$, let $\hat{\zeta}_{v}^{w}: \hat{\mathfrak{X}}_{v}^{w} \rightarrow \hat{X}_{v+1}^{w+1}$ be the maps defined by

$$
\hat{\zeta}^{w}\left(\mathbf{x}_{0}^{n}, \mathbf{y}_{0}^{n}\right)=\left(0, F_{0}\left(\mathbf{y}_{0}^{n}\right)+\sum_{j=i\left(\mathbf{y}_{0}^{n}\right)+1}^{n} F_{j}\left(\mathbf{y}_{0}^{n}\right)\right) .
$$

The map $\breve{\theta}:(\breve{\mathfrak{X}}, \breve{\mathfrak{b}}) \rightarrow(\breve{X}, \breve{b})$, defined by $\breve{\theta}_{n}=\bigoplus_{w=0}^{n} \hat{\theta}_{n+w}^{w}+\bigoplus_{w=0}^{n-1} \hat{\zeta}_{n+w}^{w}$, where $\hat{\theta}_{n+w}^{w}$ is as in Lemma A.2 is a morphism of complexes.

(4) Let $\breve{\vartheta}:(\breve{X}, \breve{b}) \rightarrow(\breve{\mathfrak{X}}, \breve{\mathfrak{b}})$ be the map induced by $\hat{\vartheta}$. It is true that $\breve{\theta} \circ \breve{\vartheta}=\mathrm{id}$ and $\breve{\vartheta} \circ$ is homotopic to the identity map. A homotopy is the family of maps $\breve{\epsilon}: \breve{\mathfrak{X}}_{n} \rightarrow \breve{\mathfrak{X}}_{n+1}$, defined by $\breve{\epsilon}_{n+1}=\bigoplus_{w=0}^{n} \hat{\epsilon}_{n+w+1}^{w}$, where $\hat{\epsilon}_{n+w+1}^{w}$ is as Lemma A.g

Proof. By Lemma A.2 we have the following special deformation retract:

$$
\bigoplus_{w \geq 0}\left(\hat{X}_{*+w}^{w}, \hat{b}\right) \stackrel{\hat{\theta}}{\stackrel{\vartheta}{\longleftrightarrow}} \bigoplus_{w \geq 0}\left(\hat{\mathfrak{X}}_{*+w}^{w}, \hat{\mathfrak{b}}\right), \quad \hat{\epsilon},
$$

where $\hat{\vartheta}=\bigoplus_{w \geq 0} \hat{\vartheta}_{*+w}^{w}, \hat{\theta}=\bigoplus_{w \geq 0} \hat{\theta}_{*+w}^{w}$ and $\hat{\epsilon}=\bigoplus_{w \geq 0} \hat{\epsilon}_{*+w}^{w}$. Consider the perturbation $\hat{\mathfrak{d}}$. Applying the perturbation lemma to this datum, we obtain a special deformation retract

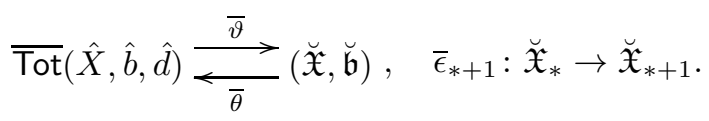

To finish the proof it remains to check that

$$
\overline{\operatorname{Tot}}(\hat{X}, \hat{b}, \hat{d})=(\breve{X}, \breve{b}), \quad \bar{\vartheta}=\breve{\vartheta}, \quad \bar{\theta}=\breve{\theta} \quad \text { and } \quad \bar{\epsilon}=\breve{\epsilon},
$$

for which it suffices to check that

$$
\hat{\theta} \circ \hat{\mathfrak{d}} \circ \hat{\vartheta}=\hat{d}, \quad \hat{\epsilon} \circ \hat{\mathfrak{d}} \circ \hat{\vartheta}=0, \quad \hat{\theta} \circ \hat{\mathfrak{d}} \circ \hat{\epsilon}=\hat{\zeta} \quad \text { and } \quad \hat{\epsilon} \circ \hat{\mathfrak{d}} \circ \hat{\epsilon}=0,
$$

where $\hat{\zeta}_{n}=\bigoplus_{w=0}^{n-1} \hat{\zeta}_{n+w}^{w}$, which follows by a direct computation.

Let $(\breve{\mathfrak{X}}, \breve{\mathfrak{b}}, \breve{\mathfrak{B}})=\operatorname{ker}\left(\left(E \otimes \bar{E}^{\otimes *}, b, B\right) \stackrel{\pi}{\longrightarrow}\left(A \otimes \bar{A}^{\otimes *}, b, B\right)\right)$.

Lemma A.4. Let $\hat{B}$ be as in Theorem 3.2. The following assertions hold:

(1) $(\hat{X}, \hat{b}, \hat{d}, \hat{B})$ is a double mixed complex.

(2) Let $(\breve{X}, \breve{b}, \breve{B})$ be the mixed complex associated with $(\hat{X}, \hat{b}, \hat{d}, \hat{B})$. The maps

$$
\breve{\vartheta}:(\breve{X}, \breve{b}, \breve{B}) \rightarrow(\breve{\mathfrak{X}}, \breve{\mathfrak{b}}, \breve{\mathfrak{B}}) \quad \text { and } \quad \breve{\theta}:(\breve{\mathfrak{X}}, \breve{\mathfrak{b}}, \breve{\mathfrak{B}}) \rightarrow(\breve{X}, \breve{b}, \breve{B}) \text {, }
$$
introduced in Lemma A.3 are morphisms of mixed complexes. 
Proof. 1) From the fact that $\hat{b} \circ \hat{b}=0$ it follows easily that $t \circ b=b \circ$. Thus we obtain that $b \circ N=N \circ b$, which implies that $\hat{b} \circ \hat{B}+\hat{B} \circ \hat{b}=0$. To prove that $\hat{d}_{\circ} \hat{B}+\hat{B} \circ \hat{d}=0$ we must check that $d^{\prime} \circ N=-N \circ d$. Let $\mathbf{x}_{0}^{n} \in X_{v}^{w}$ be an elementary tensor. Let $0=i_{0}<i_{1}<\cdots<i_{w} \leq n$ be the indices such that $x_{i_{j}} \in M$ and let $i_{w+1}=n+1$. Fix $l \in\{0, \ldots, n-1\} \backslash\left(\left\{i_{0}, \ldots, i_{w}\right\} \cup\left\{i_{1}-1, \ldots, i_{w}-1\right\}\right)$ and let $r$ such that $i_{r}<l<i_{r+1}$. A direct computation shows that

$$
t^{j}\left(F_{l}\left(\mathbf{x}_{0}^{n}\right)\right)= \begin{cases}F_{l+n+1-i_{w+1-j}} \circ t^{j}\left(\mathbf{x}_{0}^{n}\right) & \text { if } 0 \leq j \leq w-r \\ t^{\circ} F_{l+n+1-i_{r+1}} \circ t^{w-r}\left(\mathbf{x}_{0}^{n}\right) & \text { if } j=w-r+1 \\ F_{l-i_{w+2-j}} \circ t^{j-1}\left(\mathbf{x}_{0}^{n}\right) & \text { if } j>w-r+1\end{cases}
$$

Hence

$$
d^{\prime} \circ N\left(\mathbf{x}_{0}^{n}\right)=-\sum_{l=1}^{n-1} \sum_{j=0}^{w} F_{l} \circ t^{j}\left(\mathbf{x}_{0}^{n}\right)-\sum_{l=i\left(\mathbf{x}_{0}^{n}\right)+1}^{n-1} \sum_{j=0}^{w} t \circ F_{l} \circ t^{j}\left(\mathbf{x}_{0}^{n}\right)=-\sum_{j=0}^{w+1} \sum_{l=1}^{n-1} t^{j} \circ F_{l}\left(\mathbf{x}_{0}^{n}\right),
$$

as we want.

2) From Lemma A.3 we get a special deformation retract between the total complexes of the double complexes $\mathrm{BC}(\breve{X}, \breve{b}, 0)$ and $\mathrm{BC}(\breve{\mathfrak{X}}, \breve{\mathfrak{b}}, 0)$. Consider the perturbation $\breve{\mathfrak{B}}$. The result it follows by applying the perturbation lemma to this setting, and using that $\breve{B}=\breve{\theta} \circ \breve{\mathfrak{B}} \circ \breve{\vartheta}, \breve{\mathfrak{B}} \circ \breve{\epsilon}=0$ and $\breve{\epsilon} \circ \breve{\mathfrak{B}}=0$.

Proof of Theorem 3.2, It follows immediately from Lemma A.4

Proof of Proposition 3.7. It is immediate that the sequence

$$
0 \longrightarrow(\breve{\mathfrak{X}}, \breve{\mathfrak{b}}) \stackrel{i}{\longrightarrow}\left(E \otimes \bar{E}^{\otimes *}, b\right) \stackrel{\pi}{\longrightarrow}\left(A \otimes \bar{A}^{\otimes *}, b\right) \longrightarrow 0 .
$$

splits in each level via the maps $s_{n}: A \otimes \bar{A}^{\otimes n} \rightarrow E \otimes \bar{E}^{\otimes n}$ and $r_{n}: E \otimes \bar{E}^{\otimes n} \rightarrow \breve{\mathfrak{X}}_{n}$, given by $s_{n}\left(\mathbf{a}_{0}^{n}\right)=\left(a_{0}, 0\right) \otimes \cdots \otimes\left(a_{n}, 0\right)$ and $r_{n}=\mathrm{id}-s_{n} \circ \pi_{n}$. From this it follows that the connection map $\delta_{n}: \mathrm{HH}_{n}(A) \rightarrow \mathrm{HH}_{n-1}(E, M)$ is induced by the map $\delta:\left(A \otimes \bar{A}^{\otimes *}, b\right) \rightarrow(\breve{\mathfrak{X}}, \breve{\mathfrak{b}})[1]$, given by $\delta_{n}=r_{n-1}{ }^{\circ} b_{n}{ }^{\circ} s_{n}$. To finish the proof it suffices to check that $\breve{\delta}=\breve{\theta} \circ \delta$, where $\breve{\theta}:(\breve{\mathfrak{X}}, \breve{\mathfrak{b}}) \rightarrow(\breve{X}, \breve{b})$ is as in Lemma A.3

Proof of Proposition 3.8. From Proposition 3.7 and Lemma A.4 it follows that $\delta_{n}$ is induced by the morphism of complexes

$$
\delta: \operatorname{Tot}\left(\mathrm{BC}\left(A \otimes \bar{A}^{\otimes *}, b, B\right)\right) \rightarrow \operatorname{Tot}(\mathrm{BC}(\breve{X}, \breve{b}, \breve{B}))[1],
$$

given by $\hat{\delta}_{n}\left(\mathbf{a}_{0}^{n}, \mathbf{b}_{0}^{n-2}, \mathbf{c}_{0}^{n-4}, \ldots\right)=\left(\breve{\delta}_{n}\left(\mathbf{a}_{0}^{n}\right), \breve{\delta}_{n-2}\left(\mathbf{b}_{0}^{n-2}\right), \breve{\delta}_{n-4}\left(\mathbf{c}_{0}^{n-4}\right), \ldots\right)$, where $\breve{\delta}_{n}$, $\breve{\delta}_{n-2}, \breve{\delta}_{n-4}$, etcetera, are as in Proposition 3.7 To finish the proof it suffices to compose this map with the canonical projection from $\operatorname{Tot}(\mathrm{BC}(\breve{X}, \breve{b}, \breve{B}))[1]$ to $\operatorname{Tot}\left(\bar{X}_{0}, \bar{b}_{0}, \bar{d}_{0}\right)[1]$.

\section{ApPEndix B.}

Recall from the discussion above Theorem 4.3 that for each $t$-invariant element $\mathbf{x} \in X_{v}^{w}$

$$
\Upsilon(\mathbf{x})=(-\mathrm{id}+\bar{N} \circ \mathfrak{p}) \circ \sigma^{\prime} \circ d(\mathbf{x}) \in X_{v}^{w+1} .
$$

It is easy to check that $\Upsilon(\mathbf{x})$ is univocally determined by the following properties:

$$
\Upsilon(\mathbf{x}) \in \operatorname{ker}(\mathfrak{p}) \quad \text { and } \quad(x, \Upsilon(\mathbf{x})) \in P(\ddot{X}) .
$$

Let ${ }^{e} P\left(\ddot{X}_{v}^{w}\right)=\left\{(\mathbf{x}, \Upsilon(\mathbf{x})) \in \ddot{X}_{v}^{w}: \mathbf{x}\right.$ is $t$-invariant $\}$. Clearly, $P(\ddot{X})={ }^{e} P(\ddot{X}) \oplus^{t} P(\ddot{X})$. We assert that $\ddot{d}\left({ }^{e} P\left(\ddot{X}_{v}^{w}\right)\right) \subseteq{ }^{e} P\left(\ddot{X}_{v}^{w+1}\right)$. In order to prove this we will need the following result

Lemma B.1. It is true that $\bar{d} \circ \mathfrak{p}=-\mathfrak{p} \circ d^{\prime}$. 
Proof. Let $\mathbf{y}_{0}^{n} \in X_{v}^{w}$ and let $i_{w}>0$ be the last index such that $y_{i_{w}} \in M$. We have:

$$
\begin{aligned}
\mathfrak{p} \circ d^{\prime}\left(\mathbf{y}_{0}^{n}\right) & =-\sum_{j=1}^{n-1} \mathfrak{p} \circ F_{j}\left(\mathbf{y}_{0}^{n}\right)-\sum_{j=i_{w}+1}^{n-1} \mathfrak{p} \circ t_{\circ} F_{j}\left(\mathbf{y}_{0}^{n}\right) \\
& =-\sum_{j=1}^{i_{w}-2} \frac{n-i_{w}+2}{v+2}\left[F_{j}\left(\mathbf{y}_{0}^{n}\right)\right]-\sum_{j=i_{w}+1}^{n-1} \frac{n-j+1}{v+2}\left[F_{j}\left(\mathbf{y}_{0}^{n}\right)\right] \\
& -\sum_{j=i_{w}+1}^{n-1} \frac{j-i_{w}+1}{v+2}\left[t \circ F_{j}\left(\mathbf{y}_{0}^{n}\right)\right] \\
& =-\sum_{j=1}^{n-1} \frac{n-i_{w}+2}{v+2}\left[F_{j}\left(\mathbf{y}_{0}^{n}\right)\right] \\
& =-\bar{d}_{\circ} \mathfrak{p}\left(\mathbf{y}_{0}^{n}\right),
\end{aligned}
$$

where $[\mathbf{x}]$ denotes the class of $\mathbf{x} \in X_{v}^{w+1}$ in $\bar{X}_{v}^{w+1}$.

Proposition B.2. Let $\mathbf{x} \in X_{v}^{w}$ be a t-invariant element. Then

$$
\ddot{d}(\mathbf{x}, \Upsilon(\mathbf{x}))=-\frac{w+1}{w+2}\left(d^{\prime}(\mathbf{x}), \Upsilon \circ d^{\prime}(\mathbf{x})\right) .
$$

Proof. Since, by Lemma 3.3

$(t-\mathrm{id})(\Upsilon(\mathbf{x}))=d(\mathbf{x})-\frac{1}{w+2} N \circ d(\mathbf{x})=d(\mathbf{x})+\frac{1}{w+2} d^{\prime} \circ N(\mathbf{x})=d(\mathbf{x})+\frac{w+1}{w+2} d^{\prime}(\mathbf{x})$,

we have:

$$
\ddot{d}(\mathbf{x}, \Upsilon(\mathbf{x}))=\left(d(\mathbf{x})+(\mathrm{id}-t)(\Upsilon(\mathbf{x})), d^{\prime}(\Upsilon(\mathbf{x}))=\left(-\frac{w+1}{w+2} d^{\prime}(\mathbf{x}), d^{\prime}(\Upsilon(\mathbf{x}))\right) .\right.
$$

In order to finish the proof it suffices to check that

$$
\left(-\frac{w+1}{w+2} d^{\prime}(\mathbf{x}), d^{\prime}(\Upsilon(\mathbf{x}))\right) \in P(\ddot{X}) \quad \text { and } \quad \mathfrak{p}\left(d^{\prime}(\Upsilon(\mathbf{x}))=0 .\right.
$$

The first fact follows immediately from the fact that $P(\ddot{X})$ is a subcomplex of $(\ddot{X}, \ddot{b}, \ddot{d})$ and the second one follows easily from Lemma B.1

For each $v$ and $w$, let ${ }^{e} b:{ }^{e} P\left(\ddot{X}_{v}^{w}\right) \rightarrow{ }^{e} P\left(\ddot{X}_{v-1}^{w}\right)$ and ${ }^{e} \xi:{ }^{e} P\left(\ddot{X}_{v}^{w}\right) \rightarrow{ }^{t} P\left(\ddot{X}_{v-1}^{w}\right)$ be the maps defined by $\ddot{b}(\mathbf{x})={ }^{e} b(\mathbf{x})+{ }^{e} \xi(\mathbf{x})$. We now want to compute these maps. To carry out this task we will need Proposition B.3 below. Let ${ }^{t} X_{v}^{w}$ be the set of $t$-invariant elements of $X_{v}^{w}$ and let $\xi:{ }^{t} X_{v}^{w} \rightarrow{ }^{t} X_{v-1}^{w+1}$ be the map defined by

$$
\xi(\mathbf{x})=-\bar{N} \circ \mathfrak{p} \circ \sigma^{\prime} \circ d \circ b(\mathbf{x})-\bar{N} \circ \bar{b} \circ \mathfrak{p} \circ \sigma^{\prime} \circ d(\mathbf{x}) .
$$

Proposition B.3. Assume that $\mathrm{x} \in X_{v}^{w}$ is a t-invariant element. Then,

$$
{ }^{e} b(\mathbf{x}, \Upsilon(\mathbf{x}))=(b(\mathbf{x}), \Upsilon(b(\mathbf{x}))) \quad \text { and } \quad{ }^{e} \xi(\mathbf{x}, \Upsilon(\mathbf{x}))=(0, \xi(\mathbf{x})) .
$$

Proof. First note that $\ddot{b}(\mathbf{x}, \Upsilon(\mathbf{x})) \in P(\ddot{X})$, since $P(\ddot{X})$ is a subcomplex of $(\ddot{X}, \ddot{b}, \ddot{d})$. So, from the fact that

$$
\ddot{b}(\mathbf{x}, \Upsilon(\mathbf{x}))=(b(\mathbf{x}),-b(\Upsilon(\mathbf{x}))=(b(\mathbf{x}), \Upsilon(b(\mathbf{x})))+(0,-b(\Upsilon(\mathbf{x}))-\Upsilon(b(\mathbf{x}))),
$$

it follows that

$$
{ }^{e} b(\mathbf{x}, \Upsilon(\mathbf{x}))=(b(\mathbf{x}), \Upsilon(b(\mathbf{x}))) \quad \text { and } \quad{ }^{e} \xi(\mathbf{x}, \Upsilon(\mathbf{x}))=(0,-\Upsilon(b(\mathbf{x}))-b(\Upsilon(\mathbf{x}))) .
$$


To finishes the proof we must compute the last map. But, since by Proposition 3.5

$$
b \circ \sigma^{\prime} \circ d(\mathbf{x})=\sigma^{\prime} \circ b \circ d(\mathbf{x})=-\sigma^{\prime} \circ d \circ b(\mathbf{x}),
$$

we have:

$$
\begin{aligned}
-\Upsilon(b(\mathbf{x}))-b(\Upsilon(\mathbf{x})) & =(\mathrm{id}-\bar{N} \circ \mathfrak{p}) \circ \sigma^{\prime} \circ d \circ b(\mathbf{x})+b \circ(\mathrm{id}-\bar{N} \circ \mathfrak{p}) \circ \sigma^{\prime} \circ d(\mathbf{x}) \\
& =-\bar{N} \circ \mathfrak{p} \circ \sigma^{\prime} \circ d \circ b(\mathbf{x})-b \circ \bar{N} \circ \mathfrak{p} \circ \sigma^{\prime} \circ d(\mathbf{x}) \\
& =-\bar{N} \circ \mathfrak{p} \circ \sigma^{\prime} \circ d \circ b(\mathbf{x})-\bar{N} \circ \bar{b} \circ \mathfrak{p} \circ \sigma^{\prime} \circ d(\mathbf{x}),
\end{aligned}
$$

as we want.

Proof of Theorem 4.3. It is immediate that $\Lambda$ is a morphism of double mixed complexes. Using Propositions B.2 and B.3 it is easy to check that $\Psi$ is a morphism of double mixed complexes. To finish the proof it suffices to note that the maps $\Lambda$ and $\Psi$ are bijective.

We now are going to prove Proposition 4.4 To carry out this task it is convenient to first calculate $\mathfrak{p} \circ \sigma^{\prime} \circ d \circ \bar{N}$.

Lemma B.4. Let $\mathbf{x}_{0}^{n} \in X_{v}^{w}$ be an elementary tensor, let $0=i_{0}<\cdots<i_{w} \leq n$ be the indices such that $x_{i_{j}} \in M$ and let $i_{w+1}=n+1$. Given $0 \leq \alpha \leq n$, we let $j(\alpha)$ denote the number defined by $i_{j(\alpha)} \leq \alpha \leq i_{j(\alpha)+1}$. We have:

$$
\mathfrak{p} \circ \sigma^{\prime} \circ d \circ \bar{N}\left(\left[\mathbf{x}_{0}^{n}\right]\right)=\sum_{\alpha=1}^{n-1}(-1)^{\alpha} D_{\alpha}\left[F_{\alpha}\left(\mathbf{x}_{0}^{n}\right)\right],
$$

where $\left[\mathbf{x}_{0}^{n}\right]$ and $\left[F_{\alpha}\left(\mathbf{x}_{0}^{n}\right)\right]$ denote the class of $\mathbf{x}_{0}^{n}$ in $\bar{X}_{v}^{w}$ and $F_{\alpha}\left(\mathbf{x}_{0}^{n}\right)$ in $\bar{X}_{v}^{w+1}$, respectively, and

$$
D_{\alpha}=\frac{w+1}{2}-\frac{(w+1)(w+2 \alpha+2)+2(n+1)(w-j(\alpha))-2 \sum_{u=1}^{w} i_{u}}{2(w+2)(v+2)} .
$$

Proof. Let $d^{\prime \prime}=-d^{\prime}-d$. Then,

$$
d \circ \bar{N}\left(\left[\mathbf{x}_{0}^{n}\right]\right)=-d^{\prime} \circ \bar{N}\left(\left[\mathbf{x}_{0}^{n}\right]\right)-d^{\prime \prime} \circ \bar{N}\left(\left[\mathbf{x}_{0}^{n}\right]\right)=\bar{N} \circ \bar{d}\left(\left[\mathbf{x}_{0}^{n}\right]\right)-d^{\prime \prime} \circ \bar{N}\left(\left[\mathbf{x}_{0}^{n}\right]\right) .
$$

On one hand, it is immediate that

$$
\mathfrak{p} \circ \sigma^{\prime} \circ \bar{N} \circ \bar{d}\left(\left[\mathbf{x}_{0}^{n}\right]\right)=\left(\sum_{j=0}^{w} \frac{w+1-j}{w+2}\right) \mathfrak{p} \circ \bar{N} \circ \bar{d}\left(\left[\mathbf{x}_{0}^{n}\right]\right)=\frac{w+1}{2} \bar{d}\left(\left[\mathbf{x}_{0}^{n}\right]\right) .
$$

On the other hand,

$$
\mathfrak{p} \circ \sigma^{\prime} \circ d^{\prime \prime} \circ \bar{N}\left(\left[\mathbf{x}_{0}^{n}\right]\right)=\sum_{\alpha=1}^{n-1} \mathfrak{p} \circ \sigma^{\prime} \circ t^{\circ} F_{\alpha}\left(\mathbf{x}_{0}^{n}\right)=\sum_{\alpha=1}^{n-1} C_{\alpha}\left[F_{\alpha}\left(\mathbf{x}_{0}^{n}\right)\right],
$$

where

$$
\begin{aligned}
C_{\alpha} & =\frac{(w+1)\left(\alpha+1-i_{j(\alpha)}\right)}{(w+2)(v+2)}+\sum_{u=1}^{j(\alpha)} \frac{(u+w-j(\alpha))\left(i_{u}-i_{u-1}+1\right)}{(w+2)(v+2)} \\
& +\frac{(w-j(\alpha))\left(n-i_{w}+2\right)}{(w+2)(v+2)}+\sum_{u=j(\alpha)+2}^{w} \frac{(u-j(\alpha)-1)\left(i_{u}-i_{u-1}+1\right)}{(w+2)(v+2)} \\
& =\frac{(w+1)(w+2 \alpha+2)+2(n+1)(w-j(\alpha))-2 \sum_{u=1}^{w} i_{u}}{2(w+2)(v+2)} .
\end{aligned}
$$

The result follows immediately from these facts. 
Proof of Proposition 4.4, By Lemma B.4

$$
\bar{d} \circ \mathfrak{p} \circ \sigma^{\prime} \circ d \circ \bar{N}\left(\left[\mathbf{x}_{0}^{n}\right]\right)=\sum_{\alpha<\beta} L_{\alpha \beta}\left[F_{\alpha} \circ F_{\beta}\left(\mathbf{x}_{0}^{n}\right)\right],
$$

where

$$
L_{\alpha \beta}=D_{\beta}-D_{\alpha}=\frac{(w+1)(\alpha-\beta)+(n+1)(j(\beta)-j(\alpha))}{(w+2)(v+2)},
$$

and

$$
\mathfrak{p} \circ \sigma^{\prime} \circ d \circ \bar{N} \circ \bar{d}\left(\left[\mathbf{x}_{0}^{n}\right]\right)=\sum_{\alpha<\beta} L_{\alpha \beta}^{\prime}\left[F_{\alpha} \circ F_{\beta}\left(\mathbf{x}_{0}^{n}\right)\right]
$$

where

$$
\begin{aligned}
L_{\alpha \beta}^{\prime} & =\left(\frac{(w+2)(w+2 \beta+1)+2 n(w-j(\beta))-2\left(\sum i_{u}-w+j(\alpha)+\alpha\right)}{2(w+3)(v+2)}\right. \\
& \left.-\frac{(w+2)(w+2 \alpha+3)+2 n(w+1-j(\alpha))-2\left(\sum i_{u}-w+j(\beta)+2 \beta\right)}{2(w+3)(v+2)}\right) \\
& =\frac{(w+3)(\beta-\alpha)+(n-1)(j(\alpha)-j(\beta))-(v+2)}{(w+3)(v+2)} .
\end{aligned}
$$

So,

$e^{\widetilde{\varsigma}}\left(\left[\mathbf{x}_{0}^{n}\right]\right)=\frac{1}{w+1} \bar{d} \circ \mathfrak{p} \circ \sigma^{\prime} \circ d \circ \bar{N}\left(\left[\mathbf{x}_{0}^{n}\right]\right)+\frac{1}{w+2} \mathfrak{p} \circ \sigma^{\prime} \circ d \circ \bar{N} \circ \bar{d}\left(\left[\mathbf{x}_{0}^{n}\right]\right)=\sum_{\alpha<\beta} \lambda_{\alpha \beta}^{(w)}\left[F_{\alpha} \circ F_{\beta}\left(\mathbf{x}_{0}^{n}\right)\right]$,

where

as desired.

$$
\lambda_{\alpha \beta}^{(w)}=\frac{2(j(\beta)-j(\alpha))}{(w+1)(w+2)(w+3)}-\frac{1}{(w+2)(w+3)},
$$

\section{REFERENCES}

[B] D. Burghelea Cyclic homology and algebraic K-theory of spaces I, Boulder Colorado 1983, Contemp. Math., vol 55 (1986) 89-115.

[C-Q] J. Cuntz and D. Quillen Operators on noncommutative differential forms and cyclic homology, Geometry, Topology and Phisics; for Raoul Bott International Press, Cambridge MA (1995) 77-111.

[Co] G. Cortiñas On the cyclic homology of commutative algebras over arbitrary ground rings, Communications in Algebra, vol. 27:3 (1999) 1403-1412.

[C] M. Crainic On the perturbation lemma, and deformations, arXiv:Math. AT/0403266 (2004).

[G-S] M. Gerstenhaber and S. D. Schack Relative Hochschild cohomology, rigid algebras and the Bockstein, Journal Of Pure and Applied Algebra, vol 43 (1986) 53-74.

[G1] T. G. Goodwillie Relative algebraic K-theory and cyclic homology, Ann. of Math., vol 124 (1986) 347-402.

[G2] T. G. Goodwillie Cyclic homology, derivations and the free loop space, Topology, vol 24 (1985) 187-215.

[K1] L. Kadison Cyclic homology of extension algebras with application to matrix algebras, algebraic K-theory, and Nest algebras of operators, Ph. D. thesis, U. of Cal. (Berkeley) (1984).

[K2] L. Kadison A relative cyclic cohomology theory useful for computations, Cr. Acad. Sci Paris, vol 308 (1989) 569-573.

[Ka1] K. Kassel Cyclic homology, comodules and mixed complexes, Journal of Algebra, vol 107 (1987) 195-216.

[Q] D. Quillen Cyclic cohomology and algebra extensions, K-theory, vol 3 (1989) 205-246.

Departamento de Matemática, Facultad de Ciencias Exactas y Naturales, Pabellón 1 - Ciudad Universitaria, (1428) Buenos Aires, Argentina.

E-mail address: vander@dm.uba.ar

Departamento de Matemática, Facultad de Ciencias Exactas y Naturales, Pabellón 1 - Ciudad Universitaria, (1428) Buenos Aires, Argentina.

E-mail address: jjgucci@dm.uba.ar 